Document downloaded from:

http://hdl.handle.net/10251/120354

This paper must be cited as:

Amiri, A.; Cordero Barbero, A.; Darvishi, M.; Torregrosa Sánchez, JR. (2018). Stability analysis of a parametric family of seventh-order iterative methods for solving nonlinear systems. Applied Mathematics and Computation. 323:43-57.

https://doi.org/10.1016/j.amc.2017.11.040

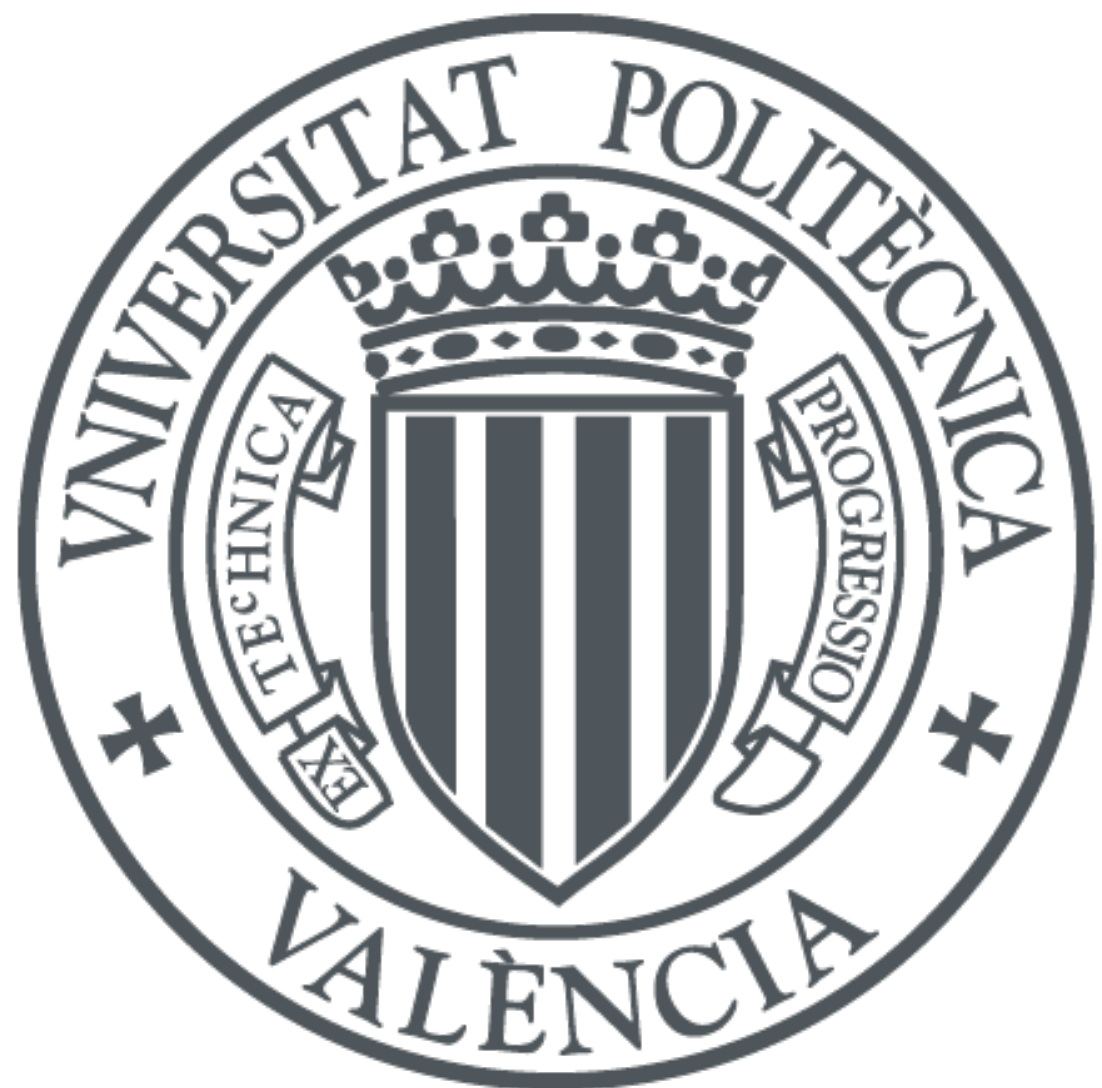

The final publication is available at

http://doi.org/10.1016/j.amc.2017.11.040

Copyright Elsevier

Additional Information 


\title{
Stability analysis of a parametric family of seventh-order iterative methods for solving nonlinear systems th
}

\author{
Abdolreza Amiri ${ }^{1}$, Alicia Cordero ${ }^{1}$, M. Taghi Darvishi ${ }^{1}$, Juan R. Torregrosa $^{1}{ }^{,}$ \\ ${ }^{a}$ Department of Mathematics, Razi University, Iran \\ ${ }^{b}$ Instituto de Matemáticas Multidisciplinar, Universitat Politècnica de València, Spain
}

\begin{abstract}
In this paper, a parametric family of seventh-order of iterative method to solve systems of nonlinear equations is presented. Its local convergence is studied and quadratic polynomials are used to investigate its dynamical behavior. The study of the fixed and critical points of the rational function associated to this class allows us to obtain regions of the complex plane where the method is stable. By depicting parameter planes and dynamical planes we obtain complementary information of the analytical results. These results are used to solve some nonlinear problems.
\end{abstract}

Keywords: Nonlinear system of equations, iterative method, stability, basin of attraction, dynamical plane

\section{Introduction}

Nonlinear systems arise in different areas of scientific computing and engineering computations, many problems modeled in science and applied problems are in the form of equations or systems of nonlinear equations. So, the analysis of these type of equations is an interesting field of study. Let us consider the system of nonlinear 5 equations $F(x)=0$, where $F: D \subseteq \mathbb{R}^{n} \longrightarrow \mathbb{R}^{n}$. As nonlinear systems are difficult to solve, the solution $\bar{x}$ is usually approximated by a fixed point function $G: D \subseteq \mathbb{R}^{n} \longrightarrow \mathbb{R}^{n}$ defining a fixed point iteration scheme. There are many root-finding iterative schemes to solve systems of nonlinear equations. The famous second order Newton's method, is a powerful iterative scheme for solving nonlinear equations and systems. But, in recent years, some researchers have proposed new iterative methods with higher order and better efficiency as an alternative to classical Newton's scheme.

Sharma and Arora in [? ] proposed an eighth-order method that is a three step scheme, denoted by $\mathrm{NM}_{8}$, as follows

$$
\begin{aligned}
y^{(k)} & =x^{(k)}-\left[F^{\prime}\left(x^{(k)}\right)\right]^{-1} F\left(x^{(k)}\right), \\
z^{(k)} & =y^{(k)}-\left[\frac{13}{4} I-G\left(x^{(k)}\right)\left(\frac{7}{2} I-\frac{5}{4} G\left(x^{(k)}\right)\right)\right]\left[F^{\prime}\left(x^{(k)}\right)\right]^{-1} F\left(y^{(k)}\right), \\
x^{(k+1)} & =z^{(k)}-\left[\frac{7}{2} I-G\left(x^{(k)}\right)\left(4 I-\frac{3}{2} G\left(x^{(k)}\right)\right)\right]\left[F^{\prime}\left(x^{(k)}\right)\right]^{-1} F\left(z^{(k)}\right)
\end{aligned}
$$

where $G\left(x^{(k)}\right)=\left[F^{\prime}\left(x^{(k)}\right)\right]^{-1} F^{\prime}\left(y^{(k)}\right)$ and $I$ is the identity matrix.

In [? ], Cordero et al. designed two eighth-order methods that the first one is describe as

$$
\begin{aligned}
y^{(k)} & =x^{(k)}-\left[F^{\prime}\left(x^{(k)}\right)\right]^{-1} F\left(x^{(k)}\right), \\
z^{(k)} & =y^{(k)}-\left[\frac{5}{4} I-\frac{1}{2}\left[F^{\prime}\left(y^{(k)}\right)\right]^{-1} F^{\prime}\left(x^{(k)}\right)+\frac{1}{4}\left(\left[F^{\prime}\left(y^{(k)}\right)\right]^{-1} F^{\prime}\left(x^{(k)}\right)\right)^{2}\right]\left[F^{\prime}\left(y^{(k)}\right)\right]^{-1} F\left(y^{(k)}\right), \\
x^{(k+1)} & =z^{(k)}-\left[\frac{3}{2} I-\left[F^{\prime}\left(y^{(k)}\right)\right]^{-1} F^{\prime}\left(x^{(k)}\right)+\frac{1}{2}\left(\left[F^{\prime}\left(y^{(k)}\right)\right]^{-1} F^{\prime}\left(x^{(k)}\right)\right)^{2}\right]\left[F^{\prime}\left(y^{(k)}\right)\right]^{-1} F\left(z^{(k)}\right) .
\end{aligned}
$$

\footnotetext{
This research was partially supported by Ministerio de Economía y Competitividad MTM2014-52016-C2-2-P and by Generalitat Valenciana PROMETEO/2016/089.

*Corresponding author
} 


$$
\begin{aligned}
y^{(k)} & =x^{(k)}-\left[F^{\prime}\left(x^{(k)}\right)\right]^{-1} F\left(x^{(k)}\right), \\
z^{(k)} & =y^{(k)}-\left[\frac{1}{4} I+\frac{1}{2}\left[F^{\prime}\left(y^{(k)}\right)\right]^{-1} F^{\prime}\left(x^{(k)}\right)+\frac{1}{4}\left(\left[F^{\prime}\left(y^{(k)}\right)\right]^{-1} F^{\prime}\left(x^{(k)}\right)\right)^{2}\right]\left[F^{\prime}\left(x^{(k)}\right)\right]^{-1} F\left(y^{(k)}\right), \\
x^{(k+1)} & =z^{(k)}-\left[\frac{1}{2} I+\frac{1}{2}\left(\left[F^{\prime}\left(y^{(k)}\right)\right]^{-1} F^{\prime}\left(x^{(k)}\right)\right)^{2}\right]\left[F^{\prime}\left(x^{(k)}\right)\right]^{-1} F\left(z^{(k)}\right) .
\end{aligned}
$$

According to Ostrowski's efficiency index, defined as $I=p^{1 / d}$, (see [? ]) where $p$ is the order of convergence and $d$ is the number of function evaluations per iteration, all these methods show better efficiency than Newton's scheme.

But, increasing order of convergence usually makes decreasing the radius of convergence, and since determining the regions where the initial guess shows better convergence behavior is important, so the study of dynamical behavior of the iterative methods is very helpful. Dynamical analysis of iterative methods for nonlinear systems is so complicated (see [? ? ]) and most of the times is impossible, but the study of the behavior of the iterative method in scalar case and in the complex plane is an interesting field of study. Moreover even in scalar case for higher order methods studying dynamical behavior is a difficult task and sometimes is impossible due to the high degree of the rational functions involved. The advent of computers in last decades, made it practically possible to study the structure of the dynamical and parameter planes of iterative methods closely in some special cases, since large amount of computational power is needed to obtain their precise shape, that can be easily performed in computers.

The main aim of this analysis is finding the regions in the complex plane where our function shows better performance when converges to the zeros of the function. But even in the scalar case, finding stable regions for a high order iterative method is not easy. High order iterative methods, even for simple scalar nonlinear function $f(z)$, usually results in a high degree fixed point operator, since the key of stability analysis is the study of the fixed point operator.

In this paper, we propose the following family of seventh-order iterative methods to solve systems of nonlinear equations, whose iterative expression is

$$
\begin{gathered}
y^{(k)}=x^{(k)}-\left[F^{\prime}\left(x^{(k)}\right)\right]^{-1} F\left(x^{(k)}\right), \\
z^{(k)}=y^{(k)}-\frac{1}{\beta}\left[F^{\prime}\left(x^{(k)}\right)\right]^{-1} F\left(y^{(k)}\right), \\
w^{(k)}=z^{(k)}-\left[F^{\prime}\left(x^{(k)}\right)\right]^{-1}\left((2-1 / \beta-\beta) F\left(y^{(k)}\right)+\beta F\left(z^{k}\right)\right), \\
x^{(k+1)}=w^{(k)}-G\left(t^{(k)}\right)\left[F^{\prime}\left(x^{(k)}\right)\right]^{-1} F\left(w^{(k)}\right),
\end{gathered}
$$

where $t^{(k)}=I-\frac{1}{\beta}\left[F^{\prime}\left(x^{(k)}\right)\right]^{-1}\left[y^{(k)}, z^{(k)} ; F\right]$ and $G: \mathbb{R}^{n \times n} \longrightarrow \mathbb{R}^{n \times n}$ is a matrix weight function that is chosen in order to obtain the seventh-order of convergence. Also $[., . ; F]: \Omega \times \Omega \subset \mathbb{R}^{n} \times \mathbb{R}^{n} \longrightarrow \mathcal{L}\left(\mathbb{R}^{n}\right)$ is the divided difference operator of $F$ on $\mathbb{R}^{n}$, defined as (see [? ])

$$
[x, y ; F](x-y)=F(x)-F(y), \text { for any } x, y \in \Omega .
$$

Let us remark that the first three steps correspond to a fourth-order parametric family whose convergence and stability was analyzed in [? ]. In this paper, we are going to analyze the dynamical behavior of class (??) on scalar functions. This analysis will be made on quadratic polynomial, as they are the simplest nonlinear functions, and it will give us important information about the stability of the family in terms of the value of the parameter and its dependence on the initial estimations used.

The rational function associated with a subclass of (??) on the quadratic polynomial $p(z)=(z-a)(z-b)$ is used in the following and denoted by $O_{p}(z)$. The obtained results can be extrapolated, up to some extent, to more complicated nonlinear function, as can be observed in related research [? ? ? ? ? ? ] and in our numerical tests.

Now, we recall some dynamical concepts that we use in this paper (see $[?]$ ). Let $R: \widehat{\mathbb{C}} \longrightarrow \widehat{\mathbb{C}}$ be a rational function, where $\widehat{\mathbb{C}}$ is the Riemann sphere, the orbit of a point $z_{0} \in \widehat{\mathbb{C}}$ is defined as:

$$
\left\{z_{0}, R\left(z_{0}\right), R^{2}\left(z_{0}\right), \ldots, R^{n}\left(z_{0}\right), \ldots\right\} \text {. }
$$


where $R^{k}$ denotes the k-th composition of the map $R$ with itself. We analyze the phase plane of the map $R$ by classifying the starting points from the asymptotic behavior of the orbits. The point $z_{0}$ is called a fixed point if $R\left(z_{0}\right)=z_{0}$ and is a periodic point of period $p>1$ if $R^{p}\left(z_{0}\right)=z_{0}$ and $R^{k}\left(z_{0}\right) \neq z_{0}$, for $k<p$. A pre-periodic point is a point $z_{0}$ that is not periodic but there exists $k>0$ such that $R^{k}\left(z_{0}\right)$, is periodic. Point $z_{0}$ is a critical point if the derivative of the rational function at $z_{0}$ vanishes, that is $R^{\prime}\left(z_{0}\right)=0$. Moreover, a fixed point $z_{0}$ is called an attracting if $\left|R^{\prime}\left(z_{0}\right)\right|<1$, superattractive if $\left|R^{\prime}\left(z_{0}\right)\right|=0$, repulsive if $\left|R^{\prime}\left(z_{0}\right)\right|>1$ and parabolic if $\left|R^{\prime}\left(z_{0}\right)\right|=1$. The fixed and critical points that are not the roots of the polynomial $p(z)$ are called strange fixed and free critical points, respectively.

The basin of attraction of an attractor $\alpha$ is defined as:

$$
\mathcal{A}(\alpha)=\left\{z_{0} \in \widehat{\mathbb{C}}: R^{n}\left(z_{0}\right) \rightarrow \alpha, n \rightarrow \infty\right\} .
$$

The Fatou set of the rational function $R$, is the set of points $z \in \widehat{\mathbb{C}}$ whose orbits tend to an attractor (fixed point, periodic point or infinity). Its complement in $\widehat{\mathbb{C}}$ is the Julia set, $\mathcal{J}(R)$. So the basin of attraction of any fixed point belongs to the Fatou set and the boundaries of these basin of attraction belong to the Julia set.

The rest of paper is organized as follows: in Section 2 the local convergence of the iterative class (??) is presented. In Section 3, we obtain the operator $O_{p}(z)$ of a particular subfamily of (??) on $p(z)$ and then the stability of fixed points of the operator is studied. Critical points of $O_{p}(z)$ are investigated in Section 4 and the parameter and dynamic planes for some members of the subclass are studied in this section. Finally, in Section 5 some of these family members are used to solve systems of nonlinear equations and the numerical stability of different members are checked.

\section{Convergence analysis}

In this section we present a local convergence theorem for family (??). In order to get this result, we introduce the following notation.

Let us denote by $X=\mathbb{R}^{n \times n}$ the space of all $n \times n$ real matrices; the weight function in this context is $G: X \rightarrow X$ such that

(i) $G^{\prime}(u)(v)=G_{1} u v$, being $G^{\prime}$ the first derivative of $G, G^{\prime}: X \rightarrow \mathcal{L}(X), G_{1} \in \mathbb{R}$ and $\mathcal{L}(X)$ denotes the space of linear mappings from $X$ to itself.

(ii) $G^{\prime \prime}(u, v)(w)=G_{2} u v w$, being $G^{\prime \prime}$ the second derivative of $G, G^{\prime \prime}: X \times X \rightarrow \mathcal{L}(X)$ and $G_{2} \in \mathbb{R}$.

As the variable of the weight function $G$ involved in class $(? ?)$ is $t^{(k)}=I-\frac{1}{\beta}\left[F\left(x^{(k)}\right)\right]^{-1}\left[y^{(k)}, z^{(k)} ; F\right]$ and it tends to $\left(1-\frac{1}{\beta}\right) I$ when $k \rightarrow \infty$, then Taylor expansion of $G$ around $\left(1-\frac{1}{\beta}\right) I$ gives

$$
G\left(t^{(k)}\right) \approx G\left(\left(1-\frac{1}{\beta}\right) I\right)+G_{1}\left(t^{(k)}-\left(1-\frac{1}{\beta}\right) I\right)+\frac{1}{2} G_{2}\left(t^{(k)}-\left(1-\frac{1}{\beta}\right) I\right)^{2} .
$$

We also use the Taylor development of the divided difference in the proof of the convergence theorem of family (??); it is obtained by Genochi-Hermite formula (see [? ]),

$$
[x, y ; F]=\int_{0}^{1} F^{\prime}(x+t(x-y)) d t, \text { for all }(x, y) \in \Omega \times \Omega \subset \mathbb{R}^{n} \times \mathbb{R}^{n}
$$

and, by developing $F^{\prime}(x+t h)$ in Taylor series around $x$, we obtain

$$
\left.\int_{0}^{1} F^{\prime}(x+t h)\right) d t=F^{\prime}(x)+\frac{1}{2} F^{\prime \prime}(x) h+\frac{1}{6} F^{\prime \prime \prime}(x) h^{2}+O\left(h^{3}\right) .
$$

Denoting by $e=x-\bar{x}$ and assuming that $F^{\prime}(\bar{x})$ is nonsingular, we have:

$$
\begin{aligned}
F(x) & =F^{\prime}(\bar{x})\left(e+C_{2} e^{2}+C_{3} e^{3}+C_{4} e^{4}+C_{5} e^{5}+O\left(e^{6}\right)\right) \\
F^{\prime}(x) & =F^{\prime}(\bar{x})\left(I+2 C_{2} e+3 C_{3} e^{2}+4 C_{4} e^{3}+5 C_{5} e^{4}+O\left(e^{5}\right)\right) \\
F^{\prime \prime}(x) & =F^{\prime}(\bar{x})\left(2 C_{2}+6 C_{3} e+12 C_{4} e^{2}+20 C_{5} e^{3}+O\left(e^{4}\right)\right) \\
F^{\prime \prime \prime}(x) & =F^{\prime}(\bar{x})\left(6 C_{3}+24 C_{4} e+60 C_{5} e^{2}+O\left(e^{3}\right)\right)
\end{aligned}
$$


where $C_{q}=\frac{1}{q !}\left[F^{\prime}(\bar{x})\right]^{-1} F^{(q)}(\bar{x}), q \geq 2$. Replacing these developments in the formula of Gennochi-Hermite and denoting the second point of the divided difference by $y=x+h$ and the error at the first step by $e_{y}=y-\bar{x}$, we have

$$
[x, y ; F]=F^{\prime}(\bar{x})\left[I+C_{2}\left(e_{y}+e\right)+C_{3}\left(e_{y}^{2}+e_{y} e+e^{2}\right)\right]+O\left(e_{y}\right)
$$

In particular, if $y$ is an approximation of the equation provided by the Newton's method, i.e. $h=x-y=$ $\left[F^{\prime}(x)\right]^{-1} F(x)$, we obtain

$$
[x, y ; F]=F^{\prime}(\bar{x})\left[I+C_{2} e+\left(C_{2}^{2}+C_{3}\right) e^{2}\right]+O\left(e^{3}\right) .
$$

The following result establishes that the class (??) has order of convergence at least seven, reaching eighthorder for a special value of parameter $\beta$.

Theorem 2.1. Let $F: D \subseteq \mathbb{R}^{n} \longrightarrow \mathbb{R}^{n}$ be a sufficiently differentiable function in an open convex set $D$ and ${ }_{90}$ let $\bar{x} \in D$ be a solution of the system of nonlinear equations $F(x)=0$. We suppose that $F^{\prime}(x)$ is continuous and nonsingular at $\bar{x}$. Then, sequence $\left\{x^{(k)}\right\}_{k \geq 0}$, obtained by expression (??), converges to $\bar{x}$ with order of convergence at least seven when $G_{0}=G\left(\left(1-\frac{1}{\beta}\right) I\right)=I, G_{1}=\beta$ and $G_{2}=\frac{1}{2}\left(-\beta+6 \beta^{2}\right)$, being in this case the error equation

$$
e^{(k+1)}=\frac{-1+5 \beta}{\beta^{3}}\left[\left(\frac{1}{\beta^{2}}+\frac{6}{\beta}-20\right) C_{2}^{5}+\left(\frac{-1+2 \beta}{2 \beta}\right) C_{2}^{4} C_{3}+\left(\frac{3-6 \beta}{2 \beta}\right) C_{2}^{3} C_{3} C_{2}\right] e^{(k)^{7}}+O\left(e^{(k)^{8}}\right)
$$

Moreover, the particular member of the family, corresponding to $\beta=\frac{1}{5}$ has order of convergence eight.

Proof. The proof of this result is based in Taylor expansion of the different elements of the iterative scheme (??). So, the expansion of $F(x)$ and $F^{\prime}(x)$ about $\bar{x}$ give

$$
F\left(x^{(k)}\right)=F^{\prime}(\bar{x})\left[e^{(k)}+C_{2} e^{(k)^{2}}+C_{3} e^{(k)^{3}}+C_{4} e^{(k)^{4}}+C_{5} e^{(k)^{5}}+C_{6} e^{(k)^{6}}+C_{7} e^{(k)^{7}}\right]+O\left(e^{(k)^{8}}\right),
$$

and

$$
F^{\prime}\left(x^{(k)}\right)=F^{\prime}(\bar{x})\left[I+2 C_{2} e^{(k)}+3 C_{3} e^{(k)^{2}}+4 C_{4} e^{(k)^{3}}+5 C_{4} e^{(k)^{4}}+6 C_{5} e^{(k)^{5}}+7 C_{6} e^{(k)^{6}}\right]+O\left(e^{(k)^{7}}\right) .
$$

We can assume that

$$
\left[F^{\prime}\left(x^{(k)}\right)\right]^{-1}=\left[I+X_{2} e^{(k)}+X_{3} e^{(k)^{2}}+X_{4} e^{(k)^{3}}+X_{5} e^{(k)^{4}}\right]\left[F^{\prime}(\bar{x})\right]^{-1}+O\left(e^{(k)^{5}}\right),
$$

where the terms of this expansion are obtained from $\left[F^{\prime}\left(x_{k}\right)\right]^{-1} F^{\prime}\left(x_{k}\right)=F^{\prime}\left(x_{k}\right)\left[F^{\prime}\left(x_{k}\right)\right]^{-1}=I$. We have

$$
X_{2}=-2 C_{2}, \quad X_{3}=4 C_{2}^{2}-3 C_{3}, \quad X_{4}=-8 C_{2}^{3}+6 C_{2} C_{3}+6 C_{3} C_{2}-4 C_{4},
$$

and, in general,

$$
X_{m}=-\sum_{j=2}^{m} j X_{m-j+1 C_{j}}, \quad m=2,3, \ldots
$$

Therefore,

$$
\begin{aligned}
y^{(k)}-\bar{x}= & C_{2} e^{(k)^{2}}+\left(2 C_{3}-2 C_{2}^{2}\right) e^{(k)^{3}}+\left(4 C_{2}^{3}-4 C_{2} C_{3}-3 C_{3} C_{2}+3 C_{4}\right) e^{(k)^{4}} \\
& +\left(-6 C_{3}^{2}-8 C_{2}^{4}+8 C_{2}^{2} C_{3}+6 C_{2} C_{3} C_{2}+6 C_{3} C_{2}^{2}-6 C_{2} C_{4}-4 C_{4} C_{2}+4 C_{5}\right) e^{(k)^{5}}+O\left(e^{(k)^{6}}\right),
\end{aligned}
$$

and it allows us to obtain

$$
\begin{aligned}
F\left(y^{(k)}\right)= & F^{\prime}(\bar{x})\left[C_{2} e^{(k)^{2}}+\left(2 C_{3}-2 C_{2}^{2}\right) e^{(k)^{3}}+\left(5 C_{2}^{3}-4 C_{2} C_{3}-3 C_{3} C_{2}+3 C_{4}\right) e^{(k)^{4}}\right. \\
& \left.+\left(-6 C_{3}^{2}-8 C_{2}^{4}+8 C_{2}^{2} C_{3}+6 C_{2} C_{3} C_{2}+6 C_{3} C_{2}^{2}-6 C_{2} C_{4}-4 C_{4} C_{2}+4 C_{5}\right) e^{(k)^{5}}\right]+O\left(e^{(k)^{6}}\right) .
\end{aligned}
$$

If we first calculate

$$
\begin{aligned}
F^{\prime}\left(x^{(k)}\right)^{-1} F\left(y^{(k)}\right)= & -C_{2} e^{(k)^{2}}+\left(-4 C_{2}^{2}+2 C_{3}\right) e^{(k)^{3}}+\left(-8 C_{2} C_{3}-6 C_{3} C_{2}+13 C_{2}^{3}+3 C_{4}\right) e^{(k)^{4}} \\
& +\left(4 C_{5}-12 C_{2} C_{4}-8 C_{4} C_{2}+26 C_{2}^{2} C_{3}+18 C_{3} C_{2}^{2}-12 C_{3}^{2}+20 C_{2} C_{3} C_{2}-38 C_{2}^{4}\right) e^{(k)^{5}}+O\left(e^{(k)^{6}}\right)
\end{aligned}
$$


and then replace these expansions in the second step of the iterative expression of (??), we get

$$
\begin{aligned}
z^{(k)}-\bar{x}= & C_{2}\left(1-\frac{1}{\beta}\right) e^{(k)^{2}}+\left(2 C_{3}-2 C_{2}^{2}-\frac{2}{\beta} C_{3}+\frac{4}{\beta} C_{2}^{2}\right) e^{(k)^{3}} \\
& +\left(C_{2}^{3}\left(4-\frac{13}{\beta}\right)-C_{2} C_{3}\left(4-\frac{8}{\beta}\right)-C_{3} C_{2}\left(3-\frac{6}{\beta}\right)+C_{4}\left(3-\frac{3}{\beta}\right)\right) e^{(k)^{4}}+\left(\left(\frac{12}{\beta}-6\right) C_{3}^{2}\right. \\
& +\left(\frac{38}{\beta}-8\right) C_{2}^{4}+\left(\frac{-26}{\beta}+8\right) C_{2}^{2} C_{3}+\left(\frac{-20}{\beta}+6\right) C_{2} C_{3} C_{2}+\left(\frac{-18}{\beta}+6\right) C_{3} C_{2}^{2} \\
& \left.+\left(\frac{12}{\beta}-6\right) C_{2} C_{4}+\left(\frac{8}{\beta}-4\right) C_{4} C_{2}+4\left(1-\frac{1}{\beta}\right) C_{5}\right) e^{(k)^{5}}+O\left(e^{(k)^{6}}\right)
\end{aligned}
$$

Now, by using Taylor expansion of $F\left(z^{(k)}\right)$

$$
\begin{aligned}
F\left(z^{(k)}\right)= & F^{\prime}(\bar{x})\left[\left(1-\frac{1}{\beta}\right) C_{2} e^{(k)^{2}}+\left(\left(2-\frac{2}{\beta}\right) C_{3}+\frac{2}{\beta} C_{2}^{2}\right) e^{(k)^{3}}\right. \\
& +\left(\left(5-\frac{15}{\beta}+\frac{1}{\beta^{2}}\right) C_{2}^{3}+\frac{4}{\beta} C_{2} C_{3}+\frac{3}{\beta} C_{3} C_{2}+\left(3-\frac{3}{\beta}\right) C_{4}\right) e^{(k)^{4}}+\left(\left(-\frac{8}{\beta^{2}}+\frac{50}{\beta}-12\right) C_{2}^{4}\right. \\
& +\left(\frac{-18}{\beta}+6\right) C_{3} C_{2}^{2}+\left(\frac{2}{\beta^{2}}+\frac{-30}{\beta}+10\right) C_{2}^{2} C_{3}+\left(\frac{2}{\beta^{2}}+\frac{-24}{\beta}+8\right) C_{2} C_{3} C_{2} \\
& \left.\left.+\left(\frac{12}{\beta}-6\right) C_{2} C_{4}+\left(\frac{8}{\beta}-4\right) C_{4} C_{2}+\left(4-\frac{4}{\beta}\right) C_{5}+\left(\frac{12}{\beta}-6\right) C_{3}^{2}\right) e^{(k)^{5}}\right]+O\left(e^{(k)^{6}}\right) .
\end{aligned}
$$

By denoting $A=\left[F^{\prime}\left(x^{(k)}\right)\right]^{-1}\left[\left(2-\frac{1}{\beta}-\beta\right) F\left(y^{(k)}\right)+\beta F\left(z^{(k)}\right)\right]$, we have

$$
\begin{aligned}
A= & \left(1-\frac{1}{\beta}\right) C_{2} e^{(k)^{2}}+\left(\left(2-\frac{2}{\beta}-2\right) C_{3}+\left(4-\frac{2}{\beta}\right) C_{2}^{2}\right) e^{(k)^{3}} \\
& +\left(\left(-1-\frac{12}{\beta}\right) C_{2}^{3}+\left(\frac{8}{\beta}-4\right) C_{2} C_{3}+\left(\frac{6}{\beta}-3\right) C_{3} C_{2}+\left(3-\frac{3}{\beta}\right) C_{4}\right) e^{(k)^{4}} \\
& +\left(\left(-4+\frac{8}{\beta}\right) C_{4} C_{2}+\left(-6+\frac{12}{\beta}\right) C_{2} C_{4}+\left(\frac{28}{\beta}+28\right) C_{2}^{4}+\left(\frac{-18}{\beta}-2\right) C_{2} C_{3} C_{2}\right. \\
& \left.+\left(\frac{-24}{\beta}-2\right) C_{2}^{2} C_{3}+\frac{-18}{\beta} C_{3} C_{2}^{2}+\left(\frac{-12}{\beta}+6\right) C_{3}^{2}+\left(\frac{-4}{\beta}+4\right) C_{5}\right) e^{(k)^{5}}+O\left(e_{k}^{6}\right),
\end{aligned}
$$

so we get

$$
\begin{aligned}
w^{(k)}-\bar{x}= & z^{(k)}-\bar{x}-A \\
= & \left(5-\frac{1}{\beta}\right) C_{2}^{3} e^{(k)^{4}}+\left(\left(\frac{10}{\beta}-36\right) C_{2}^{4}+\left(\frac{-2}{\beta}+8\right) C_{2} C_{3} C_{2}+\left(\frac{-2}{\beta}+10\right) C_{2}^{2} C_{3}+6 C_{3} C_{2}^{2}\right) e^{(k)^{5}} \\
& +O\left(e^{(k)^{6}}\right)
\end{aligned}
$$

and

$$
\begin{aligned}
F\left(w^{(k)}\right)= & F^{\prime}(\bar{x})\left(w^{(k)}-\bar{x}\right)+O\left(\left(w^{(k)}-\bar{x}\right)^{2}\right) \\
= & F^{\prime}(\bar{x})\left[\left(5-\frac{1}{\beta}\right) C_{2}^{3} e_{k}^{4}+\left(\left(\frac{10}{\beta}-36\right) C_{2}^{4}+\left(\frac{-2}{\beta}+8\right) C_{2} C_{3} C_{2}+\left(\frac{-2}{\beta}+10\right) C_{2}^{2} C_{3}+6 C_{3} C_{2}^{2}\right) e^{(k)^{5}}\right] \\
& +O\left(e^{(k)^{6}}\right) .
\end{aligned}
$$

In order to obtain the Taylor development of weight function $G$, we calculate

$$
\left[y^{(k)}, z^{(k)} ; F\right]=F^{\prime}\left(y^{(k)}\right)+F^{\prime \prime}\left(y^{(k)}\right)\left(z^{(k)}-y^{(k)}\right)+O\left(\left(z^{(k)}-y^{(k)}\right)^{2}\right),
$$

where

$$
\begin{aligned}
F\left(y^{(k)}\right) & =F^{\prime}(\bar{x})\left(y^{(k)}-\bar{x}\right)+\frac{F^{\prime}(\bar{x})}{2}\left(y^{(k)}-\bar{x}\right)^{2}+O\left(\left(y^{(k)}-\bar{x}\right)^{3}\right), \\
F^{\prime}\left(y^{(k)}\right) & =F^{\prime}(\bar{x})\left[I+2 C_{2}\left(y^{(k)}-\bar{x}\right)+3 C_{3}\left(y^{(k)}-\bar{x}\right)^{2}\right]+O\left(\left(y^{k}-\bar{x}\right)^{3}\right), \\
F^{\prime \prime}\left(y^{(k)}\right) & =F^{\prime}(\bar{x})\left[2 C_{2}+6 C_{3}\left(y^{(k)}-\bar{x}\right)+12 C_{4}\left(y^{(k)}-\bar{x}\right)^{2}\right]+O\left(\left(y^{(k)}-\bar{x}\right)^{3}\right) .
\end{aligned}
$$


Then,

$$
\begin{aligned}
{\left[y^{(k)}, z^{(k)} ; F\right]=} & F^{\prime}(\bar{x})\left[I+\left(2-\frac{1}{\beta}\right) C_{2}^{2} e^{(k)^{2}}\right. \\
& \left.+\left(2\left(2-\frac{1}{\beta}\right) C_{2} C_{3}+4\left(-1+\frac{1}{\beta}\right) C_{2}^{3}\right) e^{(k)^{3}}\right]+O\left(e^{(k)^{4}}\right)
\end{aligned}
$$

and expanding

$$
\begin{aligned}
t^{(k)}= & I-\frac{1}{\beta}\left[F\left(x^{(k)}\right)\right]^{-1}\left[y^{(k)}, z^{(k)} ; F\right] \\
= & \left(\frac{1-\beta}{\beta}\right) I+\frac{2 C_{2}}{\beta} e^{(k)}+\frac{1}{\beta^{2}}\left((1-6 \beta) C_{2}^{2}+3 \beta C_{3}\right) e^{(k)^{2}} \\
& +\frac{1}{\beta^{2}}\left((-6+16 \beta) C_{2}^{3}+(2-10 \beta) C_{2} C_{3}-6 \beta C_{3} C_{2}+4 \beta C_{4}\right) e^{(k)^{3}}+O\left(e^{(k)^{4}}\right) .
\end{aligned}
$$

When $k \rightarrow+\infty, t^{(k)} \rightarrow\left(1-\frac{1}{\beta}\right) I$, so if we apply Taylor expansion of $G(t)$ about $\left(1-\frac{1}{\beta}\right) I$ and use notations $G_{0}, G_{1}$ and $G_{2}$ as defined in (??), we get

$$
\begin{aligned}
x^{(k+1)}-\bar{x}= & \left(w^{(k)}-\bar{x}\right)-\left(G_{0}+G_{1}\left(t^{(k)}-\frac{\beta-1}{\beta}\right)+\frac{1}{2} G_{2}\left(t_{k}-\frac{\beta-1}{\beta}\right)^{2}\right)\left[F^{\prime}\left(x^{(k)}\right)\right]^{-1} F\left(w^{(k)}\right) \\
= & {\left[\left(1-G_{0}\right) I-\frac{2 G_{1}}{\beta} C_{2} e^{(k)^{2}}+\frac{1}{\beta^{2}}\left(\left(G_{1}-6 G_{1} \beta+2 G_{2}\right) C_{2}^{2}+3 G_{1} \beta C_{3}\right) e^{(k)^{2}}\right.} \\
& +\frac{1}{\beta^{3}}\left(\left(\beta G_{1}(-3+8 \beta)+G_{2}-6 \beta G_{2}\right) C_{2}^{3}+\left(\frac{2}{\beta^{2}}-\frac{10}{\beta}\right) G_{1} C_{2} C_{3}\right. \\
& \left.\left.-6 G_{1} C_{3} C_{2}+3 G_{2} C_{2} C_{3}+3 G_{2} C_{3} C_{2}+2 G_{1} \beta^{2} C_{4}\right) e^{(k)^{3}}+O\left(e^{(k)^{4}}\right)\right]\left(w^{(k)}-\bar{x}\right) .
\end{aligned}
$$

From this product and by solving the homogeneous system of equations obtained from the coefficients of $e^{(k)^{4}}$, 115 $e^{(k)^{5}}$ and $e^{(k)^{6}}$ we get $G_{0}=1, G_{1}=\beta$ and $G_{2}=\frac{1}{2}\left(-\beta+6 \beta^{2}\right)$. So we have the desired order with error equation (??). From the error equation one can see that special case when $\beta=\frac{1}{5}$, we have an iterative scheme of order 8 .

In the previous results we imposed some conditions on the weight functions to obtain desired order. By using the obtained coefficients $G_{0}, G_{1}$ and $G_{2}$, one could use different weight functions $G(t)$, satisfying the conditions of previous theorem, for designing specific families of iterative schemes. For example,

$$
G\left(t^{(k)}\right)=I+\beta\left(t^{(k)}-\left(1-\frac{1}{\beta}\right) I\right)+\frac{1}{4}\left(-\beta+6 \beta^{2}\right)\left(t^{(k)}-\left(1-\frac{1}{\beta}\right) I\right)^{2}
$$

or the rational function,

$$
G\left(t^{(k)}\right)=I+\frac{10 \beta-1}{(1-6 \beta) \beta}\left[t^{(k)}\right]^{-1}\left[\frac{1-6 \beta}{10 \beta-1} I-\frac{(1-2 \beta) \beta}{10 \beta-1} t^{(k)}\right]
$$

The subclass corresponding to the first function $G\left(t^{(k)}\right)$ is denoted by M7, and its stability in the scalar case, in terms of dependence on initial estimation, is developed in the following sections.

\section{Fixed and critical points}

In this section, we study the fixed points of operator $O_{p}(z)$ as a function of $\beta$ and their stability, and analyze its critical points. First, we consider the fixed point operator correspond to the family (??) which we denote by $O(z)$. When this fixed point operator is applied on quadratic polynomial $p(z)=(z-a)(z-b)$, the following 
rational function $O_{p}(z)$ is obtained

$$
\begin{aligned}
O_{p}(z)= & \frac{(a-z)(b-z)}{a+b-2 z}+\frac{(a-z)^{2}(b-z)^{2}}{\beta(a+b-2 z)^{3}}+z+\frac{1}{\beta(a+b-2 z)^{7}}+(a-z)^{2}(b-z)^{2}\left(a^{4}(-1+\beta)\right. \\
& +b^{4}(-1+\beta)+2 b^{3}(4-5 \beta) z+b^{2}(-23+34 \beta) z^{2}-6 b(-5+8 \beta) z^{3}+3(-5+8 \beta) z^{4} \\
& +2 a^{3}(b(-2+3 \beta)+(4-5 \beta) z)+\cdots+1 /\left(4 \beta^{5}(a+b-2 z)^{2} 3\right)(a-z)^{4}(b-z)^{4} \\
& \left(a^{4} \beta+b^{4}(-1+5 \beta)-10 a^{3} \beta z+38 a^{2} \beta z^{2}-\cdots+2 b\left(3 a^{3} \beta-23 a^{2} \beta z+58 a \beta z^{2}\right.\right. \\
& \left.\left.+2(1-24 \beta) z^{3}\right)\right)\left(b^{4} \beta+a^{4}(-1+5 \beta)-10 b^{3} \beta z+38 b^{2} \beta z^{2}-\cdots+2(1-24 \beta) z^{3}\right) \\
& \left.\left(4 a^{8} \beta^{3}+4 b^{8} \beta^{3}+8 a^{7} \beta^{3}(5 b-9 z)-\cdots+\left(1+10 \beta-96 \beta^{2}-1920 \beta^{3}\right) z^{7}\right)\right)
\end{aligned}
$$

Since even for quadratic polynomials, $O_{p}(z)$ is so complicated and difficult to work with, instead we use conjugacy maps to get a simpler operator. By using Möbius map,

$$
M(z)=\frac{z-a}{z-b}, \quad M^{-1}(z)=\frac{z b-a}{z-1},
$$

with properties:

$$
M(\infty)=1, \quad M(a)=0, \quad M(b)=\infty,
$$

P. Blanchard in [?] proved that, for quadratic polynomials, Newton's operator is conjugated to rational function $z^{2}$, in a similar way, it is easy to show that operator $O_{p}(z)$ is conjugated to the rational function (not depending on $a$ and $b$ )

$$
M_{p}(z, \beta)=z^{7} \frac{\left(-1+5 \beta+14 \beta z+14 \beta z^{2}+6 \beta z^{3}+\beta z^{4}\right) r(z)}{\left(\beta+6 \beta z+14 \beta z^{2}+14 \beta z^{3}-z^{4}+5 \beta z^{4}\right) s(z)},
$$

where $r(z)=4 \beta^{2}-24 \beta^{3}+80 \beta^{4}+\left(\beta+26 \beta^{2}-188 \beta^{3}+900 \beta^{4}\right) z+\left(6 \beta+72 \beta^{2}-608 \beta^{3}+4592 \beta^{4}\right) z^{2}+\left(14 \beta+108 \beta^{2}-\right.$ $\left.968 \beta^{3}+13952 \beta^{4}\right) z^{3}+\left(10 \beta+128 \beta^{2}-688 \beta^{3}+28080 \beta^{4}\right) z^{4}+\left(-1+3 \beta+114 \beta^{2}+8 \beta^{3}+39580 \beta^{4}\right) z^{5}+\left(-4 \beta+44 \beta^{2}+\right.$ $\left.320 \beta^{3}+40400 \beta^{4}\right) z^{6}+\left(184 \beta^{3}+30496 \beta^{4}\right) z^{7}+\left(40 \beta^{3}+17216 \beta^{4}\right) z^{8}+\left(4 \beta^{3}+7260 \beta^{4}\right) z^{9}+2240 \beta^{4} z^{10}+480 \beta^{4} z^{11}+$ $64 \beta^{4} z^{12}+4 \beta^{4} z^{13}$ and $s(z)=4 \beta^{4}+64 \beta^{4} z+480 \beta^{4} z^{2}+2240 \beta^{4} z^{3}+\left(4 \beta^{3}+7260 \beta^{4}\right) z^{4}+\left(40 \beta^{3}+17216 \beta^{4}\right) z^{5}+$ $\left(184 \beta^{3}+30496 \beta^{4}\right) z^{6}+\left(-4 \beta+44 \beta^{2}+320 \beta^{3}+40400 \beta^{4}\right) z^{7}+\left(-1+3 \beta+114 \beta^{2}+8 \beta^{3}+39580 \beta^{4}\right) z^{8}+(10 \beta+$ $\left.128 \beta^{2}-688 \beta^{3}+28080 \beta^{4}\right) z^{9}+\left(14 \beta+108 \beta^{2}-968 \beta^{3}+13952 \beta^{4}\right) z^{10}+\left(6 \beta+72 \beta^{2}-608 \beta^{3}+4592 \beta^{4}\right) z^{11}+(\beta+$ $\left.26 \beta^{2}-88 \beta^{3}+900 \beta^{4}\right) z^{12}+\left(4 \beta^{2}-24 \beta^{3}+80 \beta^{4}\right) z^{13}$.

The fixed and critical points as well as their asymptotic behavior depend on the values of parameter $\beta$. The fixed points are the roots of equation $M_{p}(z, \beta)=z$, or $z(z-1) q(z)=0$. In this case, $q(z)$ is a polynomial of degree 22 , whose roots are different from $z=0, \infty$ and $z=1$. That is, fixed points of operator $M_{p}(z, \beta)$ are, $z=0$, and $z=\infty$, that correspond to the roots of polynomial $p(z), z=1$ and the 22 roots of $q(z)$, denoted by $s_{i}(\beta), i=1,2, \ldots, 22$. The fixed points that do not correspond to the roots of $p(z)$ are called strange fixed points.

In order to study the stability of the fixed points, we calculate the first derivative of $M_{p}(z, \beta)$ and evaluate it at every fixed point. The resulting absolute value, called stability function of the fixed point, gives us information about the asymptotic behavior of the point. As $z=0$ is the root of order 6 of $M_{p}(z, \beta)$ so $M_{p}^{\prime}(z, \beta)$ vanishes at $z=0$ and $M_{p}^{\prime}(z, \beta) \rightarrow \infty$ when $z \rightarrow \infty$ so we conclude that $z=0$ and $z=\infty$ are always superattracting fixed points. But the stability of other fixed points depend on the value of parameter $\beta$.

On the other hand, changes in the multiplicity of the fixed points imply also alterations in their dynamical behavior, as they are related with bifurcations of different kind. For different values of $\beta$ we have:

- If $\beta=0$, the method is not defined, so we neglect it.

- The stability function of $z=1$ is denoted by $S_{\text {one }}=\left|M_{p}^{\prime}(1, \beta)\right|$, where

$$
M_{p}^{\prime}(1, \beta)=\frac{\left(33554432 \beta^{5}\left(1-70 \beta+704 \beta^{2}+21760 \beta^{3}-262144 \beta^{4}+7413760 \beta^{5}\right)\right)}{\left((1-40 \beta)^{2}\left(1-30 \beta-496 \beta^{2}+1920 \beta^{3}-185344 \beta^{4}\right)^{2}\right)}
$$

By analyzing this function we can see that when $\beta \rightarrow \frac{1}{40},\left|M_{p}^{\prime}(1, \beta)\right| \rightarrow \infty$ and if $\beta \rightarrow \pm \infty,\left|M_{p}^{\prime}(1, \beta)\right|=$ 4096

905. Besides, fixed point $z=1$ is attracting in an apple shape set of complex plane that lies in a small rectangle and contains the origin, that is $\left|M_{p}^{\prime}(1, \beta)\right|<1$ (see Figure ??). 


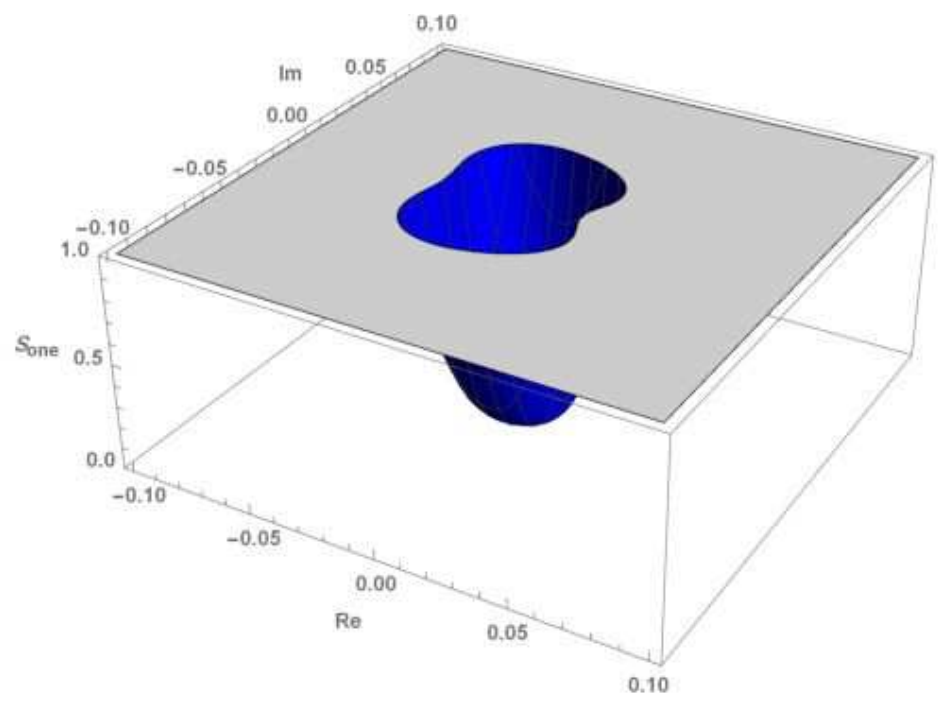

Figure 1: Attractive regions for strange fixed points $S_{\text {one }}$.

- For many different values of $\beta$ in the complex plane, strange fixed points $s_{i}(\beta), i=1,2, \cdots, 22$ are equal to 1 , so strange fixed point 1 can have different multiplicities. However, the behavior of the strange fixed points are different in the complex plane especially around 0 , as in a small region near 0 some of them satisfy $S_{i}=\left|M_{p}^{\prime}\left(s_{i}(\beta), \beta\right)\right|<1$. Figure ??, depicts $\left|M_{p}^{\prime}\left(s_{i}(\beta), \beta\right)\right|<1$, for $i=18,19, \cdots, 22$ and strange fixed point 1 simultaneously near 0 . These six strange fixed points have the bigger regions in which $\left|M_{p}^{\prime}\left(s_{i}(\beta), \beta\right)\right|<1$, in this figure $S_{\text {one }}$ denotes strange fixed point $z=1$. One can see more details of these basins of attractions in Figure ??.

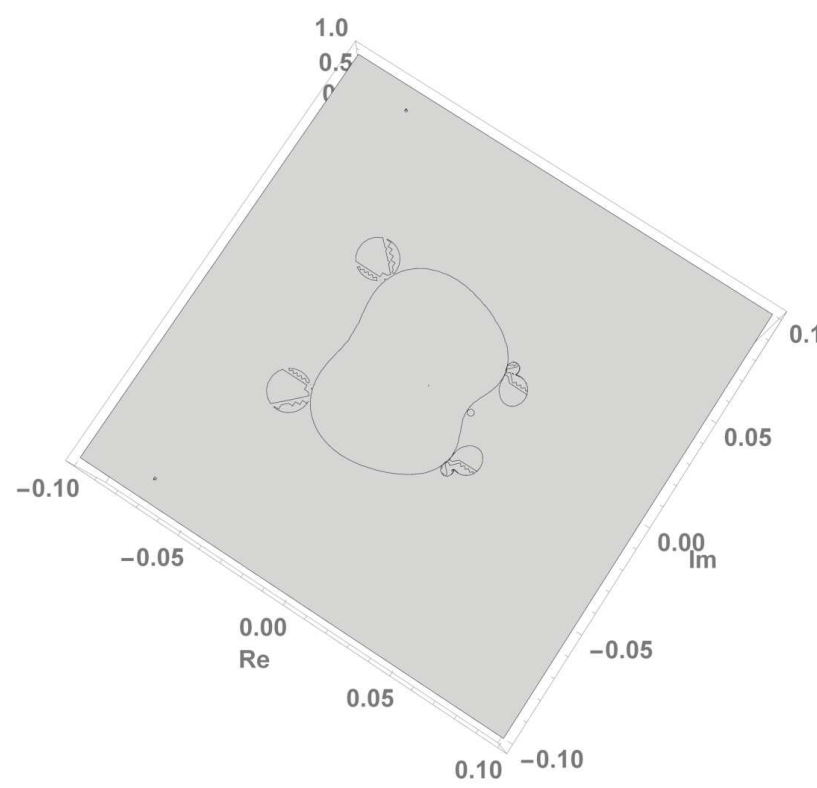

0.10

Figure 2: Some attractive regions for strange fixed points $S_{\text {one }}$ and $S_{18}, S_{19}, \cdots, S_{22}$

Figure ?? shows boundaries of regions around the basin of attraction of strange fixed point $z=1$, in which the basins of attraction of some strange fixed points lie. These five regions are denoted by $B 1, B 2, B 3$, $B 4$ and $B 5$ (see Figure ??). Boundary B1 is intersected with that of $z=1$ in $\beta \approx 0.2194+0.02447 i$ and $\beta \approx 0.02315+0.02002 i$, approximately, and boundary B2 is intersected with $z=1$ in $\beta \approx 0.2194-0.02447 i$ and $\beta \approx 0.02315-0.02002 i$. Boundaries B3 and B4 are tangent with basin of attraction of $z=1$ respectively at the points $\beta=-0.0373793-0.0286275 i$ and $\beta=-0.0373793+0.0286275 i$ and boundary B5 is tangent with the basin of attraction of $z=1$ at $\beta=0.0184166$.

By summarizing, the following result can be established. 


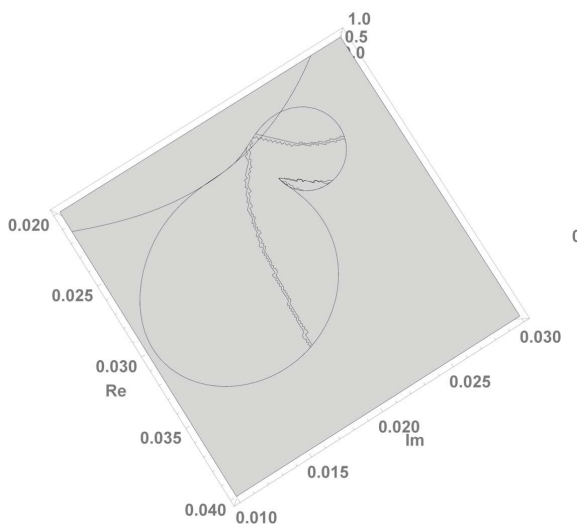

(a) $B_{1}$

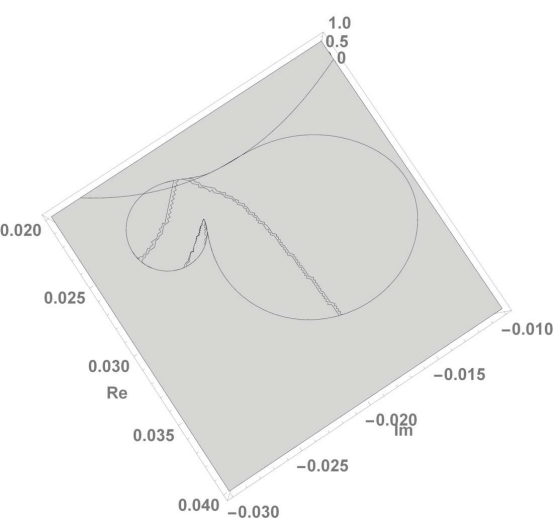

(b) $B_{2}$

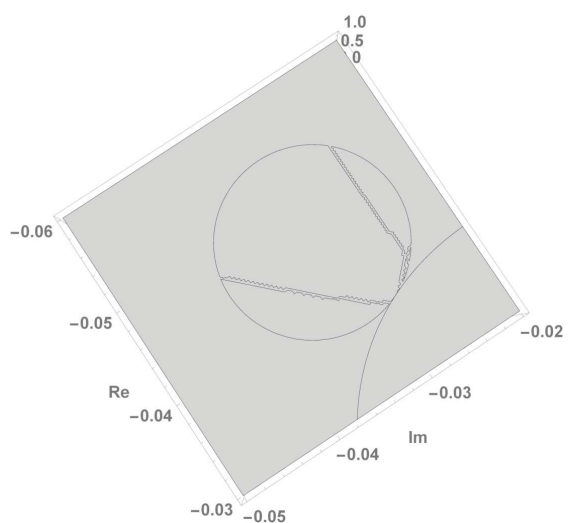

(c) $B_{3}$

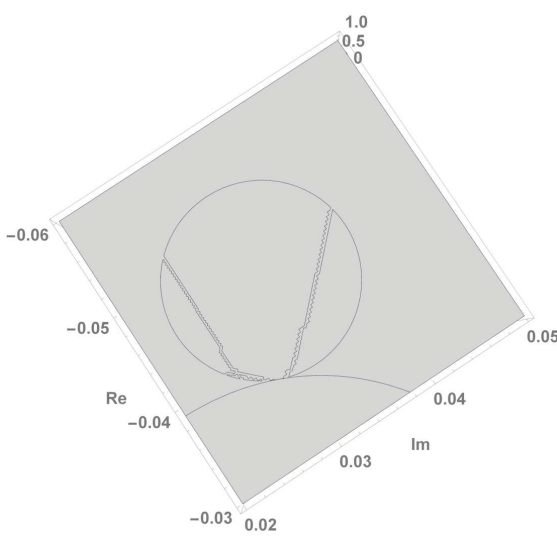

(d) $B_{4}$

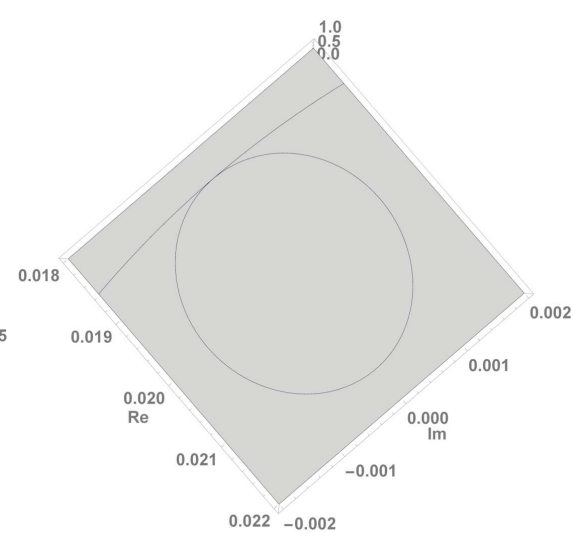

(e) $B_{5}$

Figure 3: Some attractive regions for strange fixed points $S_{\text {one }}$ and $S_{18}, S_{19}, \cdots, S_{22}$.

Proposition 3.1. Strange fixed points $s_{i}(\beta), i=1,2, \cdots, 22$ and $z=1$ satisfy the following statements:

(i) Strange fixed point $z=1$ for complex values near zero which lie in the square $[-0.04,0.025] \times$ $[-0.045,0.045]$ is attracting, but for values far from zero is repulsive.

(ii) The major attractive regions for strange fixed points $s_{i}(\beta), i=1,2, \cdots, 22$ are near zero and around the basin of attraction of strange fixed point $z=1$.

(iii) Among all strange fixed points, $s_{i}(\beta), i=18,19, \cdots, 22$ have the major attractive regions and these regions lie in the squares $[0.02,0.036] \times[0.01,0.027],[0.02,0.036] \times[-0.01,-0.027],[-0.055,-0.035] \times$ $[-0.042,-0.02]$ and $[-0.055,-0.035] \times[0.042,0.02]$.

Let us also recall that critical points of $M_{p}(z, \beta)$ are the roots of $M_{p}^{\prime}(z, \beta)=0$, since we have

$$
M_{p}^{\prime}(z, \beta)=(z+1)^{12} z^{6} u(z),
$$

where $u(z)=1400 \beta^{5}+\left(-4 \beta-40 \beta^{2}+1012 \beta^{3}-6504 \beta^{4}+18080 \beta^{5}\right) z+\left(-16 \beta+71 \beta^{2}+2506 \beta^{3}-25464 \beta^{4}+\right.$ $\left.106640 \beta^{5}\right) z^{2}+\left(-12 \beta+440 \beta^{2}+2244 \beta^{3}-51208 \beta^{4}+377120 \beta^{5}\right) z^{3}+\left(54 \beta+274 \beta^{2}+510 \beta^{3}-49668 \beta^{4}+886920 \beta^{5}\right) z^{4}+$ $\left(6-36\right.$ beta $\left.-224 \beta^{2}+2184 \beta^{3}-7824 \beta^{4}+1458240 \beta^{5}\right) z^{5}+\left(-11-42 \beta-310 \beta^{2}+4540 \beta^{3}+20592 \beta^{4}+1716960 \beta^{5}\right) z^{6}+$ $\left(6-36 \beta-224 \beta^{2}+2184 \beta^{3}-7824 \beta^{4}+1458240 \beta^{5}\right) z^{7}+\left(54 \beta+274 \beta^{2}+510 \beta^{3}-49668 \beta^{4}+886920 \beta^{5}\right) z^{8}+(-12 \beta+$ $\left.440 \beta^{2}+2244 \beta^{3}-51208 \beta^{4}+377120 \beta^{5}\right) z^{9}+\left(-16 \beta+71 \beta^{2}+2506 \beta^{3}-25464 \beta^{4}+106640 \beta^{5}\right) z^{10}+\left(-4 \beta-40 \beta^{2}+\right.$ $\left.1012 \beta^{3}-6504 \beta^{4}+18080 \beta^{5}\right) z^{11}+\left(-14 \beta^{2}+154 \beta^{3}-700 \beta^{4}+1400 \beta^{5}\right) z^{12}$. So the critical points of $M_{p}(z, \beta)$ are $z=0, z=\infty$ and $z=-1$ and the 12 roots of polynomial $u(z)$. We denote these 12 roots of $u(z)$ as $c_{i}(\beta)$, $i=1,2, \cdots 12$.

On the other hand, to obtain some properties of $c_{i}(\beta)$ we discretize the square of $[-2,2] \times[-2,2]$ in the complex plane with $400 \times 400$ mesh points. We denote this mesh points by $(i, j)$, where $i, j=1,2, \cdots, 400$, then by obtaining the roots of $p(z)$ for each $\beta=(i, j)$, a numerical value of critical points $c_{i}$ in the square $[-2,2] \times[-2,2]$, for $i=1,2, \cdots, 12$ is obtained. 
A classical result establishes that there is at least one critical point associated with each invariant Fatou component. Points $z=\infty$ and $z=0$ are both superattractive fixed points of $M_{p}(z, \beta)$, so they also are critical points and give rise to their respective Fatou components. So, the way to calculate the parameter planes associate to $M_{p}(z, \beta)$ is to study the orbits of each free independent critical point for all the complex values of the parameter $\beta$ in the defined mesh. In the next section we investigate these parameter planes of $M_{p}(z, \beta)$.

\section{The parameter space}

In this section we study the behavior of $M_{p}(z, \beta)$. As mentioned in the previous sections, the dynamical behavior of operator $M_{p}(z, \beta)$ depends on the values of the parameter $\beta$. There is at least one critical point associated with each invariant Fatou component [? ]. So, the way to calculate the parameter planes associate to $M_{p}(z, \beta)$ is the study of the orbits of each free independent critical point for all the complex values of the parameter $\beta$ in the defined mesh. In order to get the parameter plane we study the orbits of the free critical points for each $\beta$ in the complex square $[-2,2] \times[-2,2]$.

Remember regions near zero are major regions in which $\left|M_{p}^{\prime}\left(s_{i}(\beta), \beta\right)\right|<1$ and some strange fixed points are attracting near zero, so we only focus our attention in this region. To find the most stable members of the

family M7, we are looking for regions in which the orbits of free critical points converge to zero or infinity. Since 12 critical points of $M_{p}(z, \beta)$ are two-by-two dependent, so we only study the parameter plane of 6 of them. In Figure ??, the parameter planes of the 6 free independent critical points are shown. By using the routines appearing in [? ], we have painted in red the values of the parameter that makes the critical point converge to $z=0$ or $z=\infty$ (brighter as lower is the number of iterations needed), and in black if the critical point has not converged after 600 iterations, or has converged to another element (attracting strange fixed point or periodic orbit).

By comparing the parameter plane of critical points of the family (??) and Figures ?? and ?? one can see for the regions near zero, some strange fixed points are attractive and members of the family close to zero are the most unstable members of the family. In fact, there is no other pathologic behavior as attracting periodic orbits; so, the family is actually very stable.

As we mentioned in the previous section, the biggest attractive regions of the strange fixed points of the family are B1, B2, B3, B4, B5 and the corresponding of stability function $S_{\text {one }}$, so we select some members of the family in these regions or outside them to depict their dynamical planes. These planes show different behaviors of the members of family (??) (see Figure ??-??) and have been generated by iterating an element of the family (??) that is, for a constant $\beta$ and using each point of the complex plane as an initial estimation (see [? ] for the codes). These dynamical planes are built by using a mesh of $400 \times 400$ points, maximum number of iterations 1500 and a tolerance of $10^{-3}$. In this section, the basins of attraction of 0 and $\infty$ for all the dynamical planes are depicted respectively with orange and blue. Figure ?? and ?? show the dynamical planes of members of $\beta=0.018$ and $\beta=0.019$ respectively. These two members are chosen to be near $\beta=0.0184166$ where the boundaries of the circle $B 5$ and attracting region of strange fixed point $z=1$ are tangent. Let us recall that $\beta=0.018$ is in the attracting region of the strange fixed point $z=1$ and is a member of the family with three basins of attraction corresponding to fixed points $z=0, z=1$ and $z=\infty$. In this dynamical plane, the basin of attraction of $z=1$ is shown in green. On the other hand, $\beta=0.019$ is inside B5 and its respective dynamical plane has four basins of attraction, that correspond to $z=0, z=\infty$ and two strange fixed points whose values at $\beta=0.019$ are $z=0.995133-0.0985444 i$ and $z=0.995133+0.0985444 i$ and their basins of attraction are shown respectively with green and red.

Figure ?? and ?? respectively show the dynamical plane of $\beta=-0.037-0.028 i$ and $\beta=-0.037+0.028 i$ that are in the region where $z=1$ is attracting and near the points $\beta \approx-0.0373793-0.0286275 i$ and $\beta \approx$ $-0.0373793+0.0286275 i$ in which the basin of attraction of $z=1$ is tangent with regions B3 and B4. So, for each of them there are three basins of attraction $z=0, z=1$ and $z=\infty$. Basin of attractions for $z=1$ is shown with green color.

Figures ?? and ?? show the dynamical planes associated to two values of $\beta$ that are inside B5 and B6 and each of them has four basins of attraction. Two of them correspond to 0 and $\infty$ and the other ones correspond to the other two attracting strange fixed points. The values of these strange fixed points at $\beta=-0.039+0.028 i$ are $z=0.8348881+0.232033 i$ and $z=1.11189-0.309021 i$ and their respective basins are shown respectively with green and red color. Moreover, the values of two strange fixed points at $\beta=-0.039-0.028 i$ are $z=$ $0.8348881-0.232033 i$ and $z=1.11189+0.309021 i$ and their basins are shown respectively with green and red color.

On the other side, Figures ?? to ?? are related to the points that are inside and outside regions B1 and B2. Let us remark that B1 and B2 are not tangent, but secant, with $S_{\text {one }}$. Area B1 is intersected with $S_{\text {one }}$ at $\beta \approx 0.2194+0.02447 i$ and $\beta \approx 0.02315+0.02002 i$ and $\mathrm{B} 2$ is intersected with $S_{\text {one }}$ at $\beta \approx 0.2194-0.02447 i$ and $\beta \approx 0.02315-0.02002 i$. So, we choose three points for each of them to show the properties of the members of 


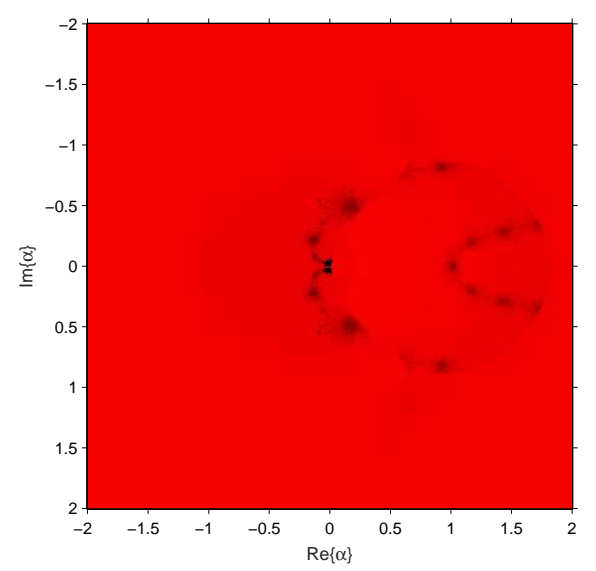

(a) $c_{1}$

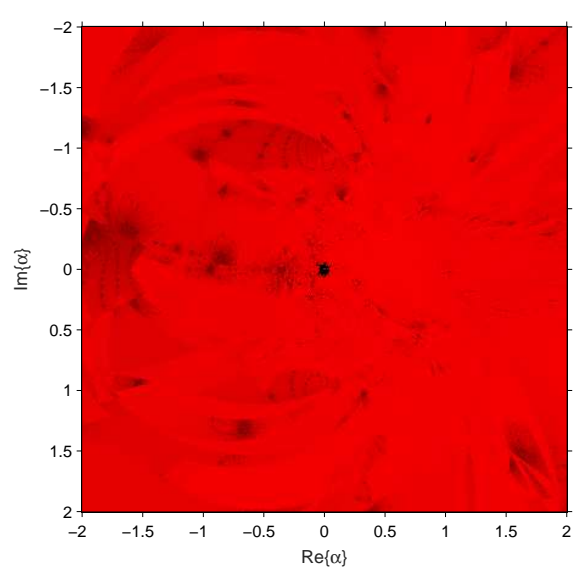

(c) $c_{3}$

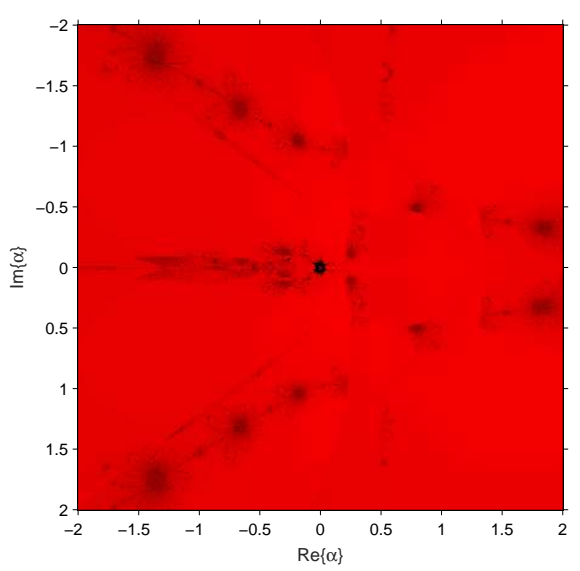

(e) $c_{5}$

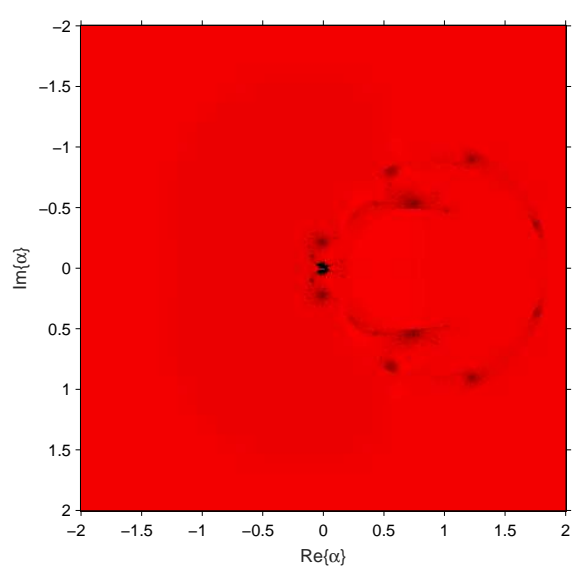

(b) $c_{2}$

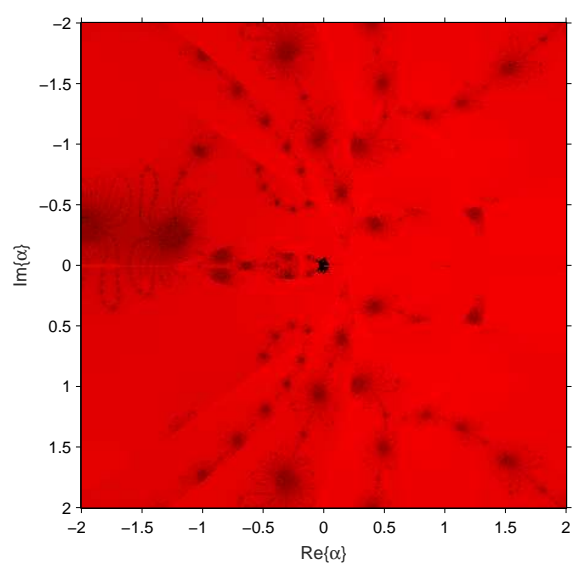

(d) $c_{4}$

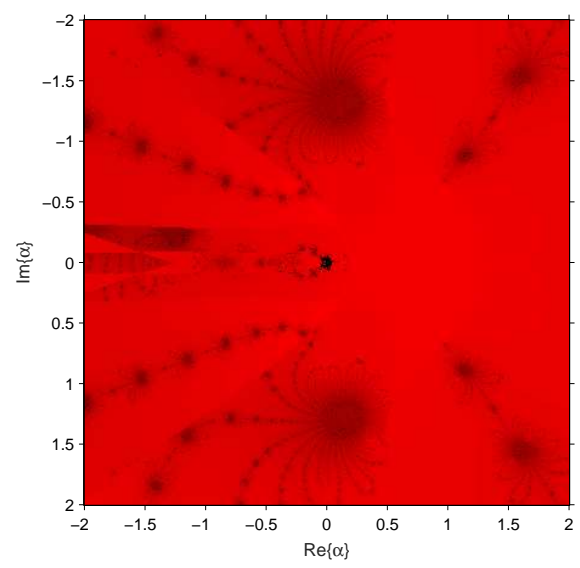

(f) $c_{6}$

Figure 4: Parameter plane for critical points $c_{1}, c_{2}, \cdots, c_{6}$

the family that lie inside or outside of these regions. Values $\beta=0.023-0.019 i$ and $\beta=0.023+0.019 i$ which are only in $S_{\text {one }}$, so have only three basins of attractions (see Figure ?? and ??).

Figures (??) and (??) show the dynamical behavior of the members of the class of iterative methods corresponding to $\beta=0.023-0.021 i$ and $\beta=0.023+0.021 i$ of the family that lie in the overlapping regions of B1 and $\mathrm{B} 2$ with $S_{\text {one }}$ and have five basins of attraction, that for $\beta=0.023+0.021 i$ are $z=0, z=\infty$ and $z=1$, and those of two strange fixed points whose at $\beta=0.023-0.019 i$ are $z=0.839234-0.125821 i$ and $z=1.16537+0.174716 i$ 


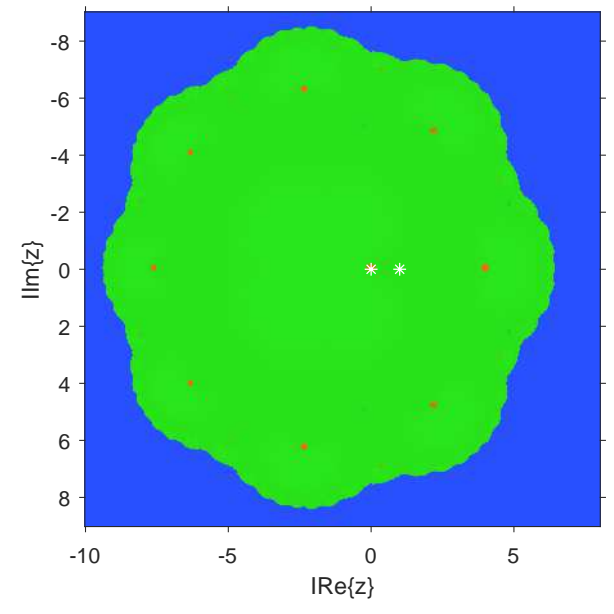

(a) $\beta=0.018$

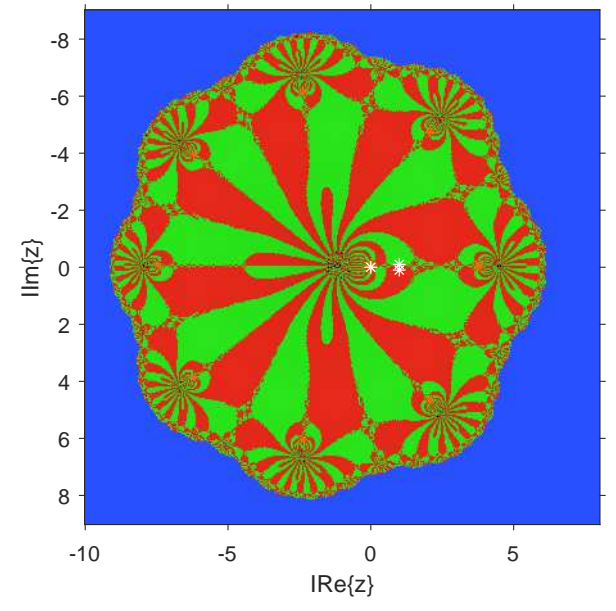

(b) $\beta=0.019$

Figure 5: Dynamical planes of $\beta=0.018$ and $\beta=0.019$.

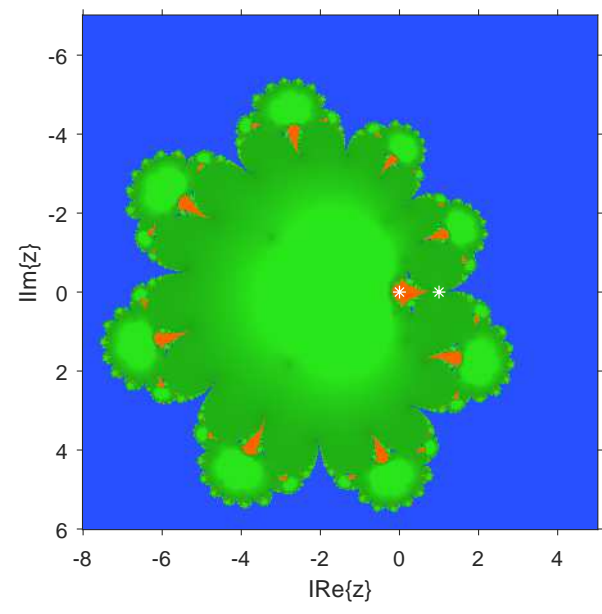

(a) $\beta=-0.037-0.028 i$

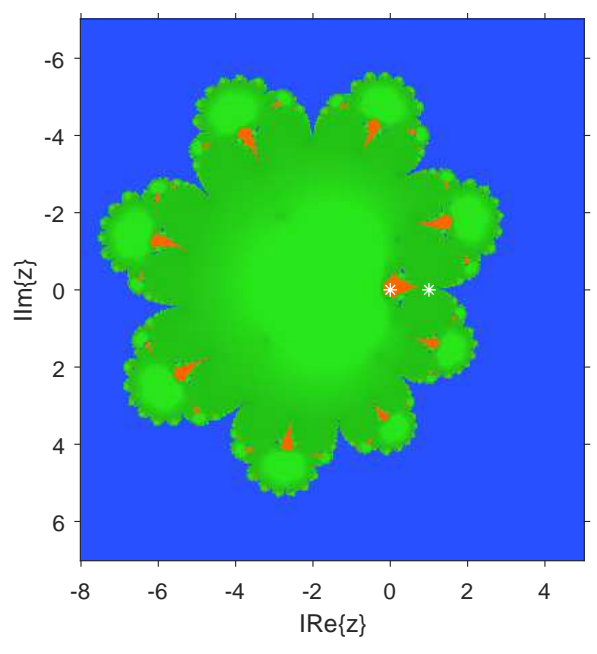

(b) $\beta=-0.037+0.028 i$

Figure 6: Dynamical planes of $\beta=-0.037-0.028 i$ and $\beta=-0.037+0.028 i$.

(their basins are shown respectively with green and red color). Also for $\beta=0.023-0.021 i$ the basins of attraction correspond to $z=0, z=\infty$ and two strange fixed point whose values are $z=0.839234+0.125821 i$ and $z=1.16537-0.174716 i$ (they are shown respectively with green and red colors). For both $\beta=0.023-0.019 i$ and $\beta=0.023+0.019 i$, the basin of attraction of $z=1$ is shown with grey color (when after few iterations converges to $z=1$ ) and black (when after many iterations converges to $z=1$ ).

Figures (??) and (??) are related to $\beta=0.024+0.021$ and $\beta=0.024-0.021$ that are inside B1 and B2, but not in the overlapping region with $S_{\text {one }}$. So, for both of them there are four basins of attraction: those corresponding to $z=0$ and $z=\infty$ and, in case of $\beta=0.024+0.021$, two strange fixed points $z=1.29489+0.0781489 i$ and $z=0.769466-0.0464388 i$ (their basins of attraction are shown respectively with green and red color); for $\beta=0.024-0.021$, the strange fixed points are $z=1.29489-0.0781489 i$ and $z=0.769466+0.0464388 i$ and their basins of attraction are shown also with green and red color.

Figures ??) to (?? show the members $\beta=0.1-0.1 i, \beta=0.5+0.1 i, \beta=5+5 i$ and $\beta=30+30 i$ of the family that all are far from 0 and are chosen randomly. They are in the regions with no attracting strange fixed points. So, there are only two basins of attraction corresponding to $z=0$ and $z=\infty$.

In the next section, we use some of these family members to solve systems of nonlinear equations and check the obtained values, observing their numerical stability and comparing it with the qualitative information. 


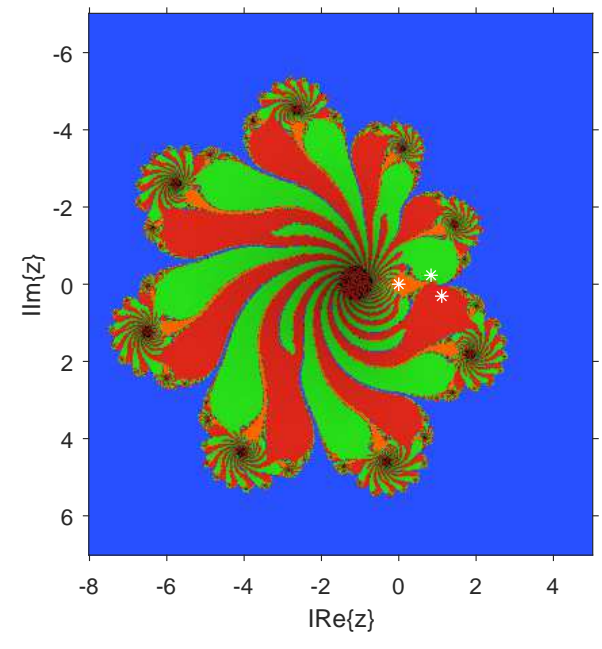

(a) $\beta=-0.039-0.028 i$

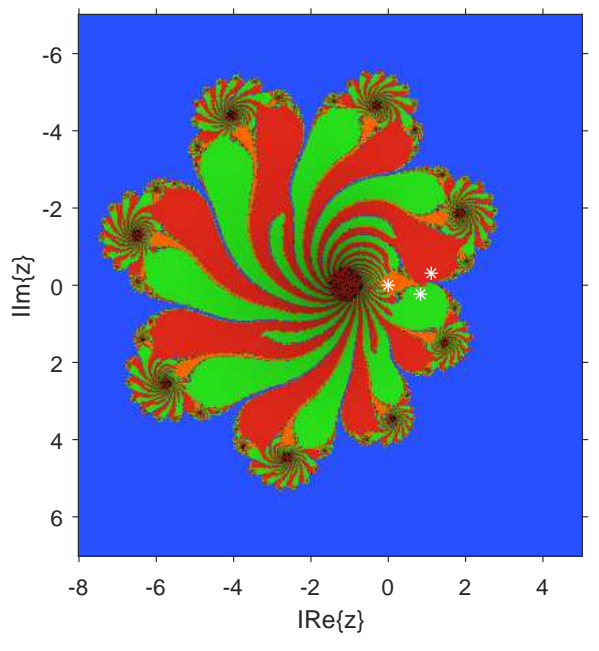

(b) $\beta=-0.039+0.028 i$

Figure 7: Dynamical planes of $\beta=-0.039-0.028 i$ and $\beta=-0.039+0.028 i$.

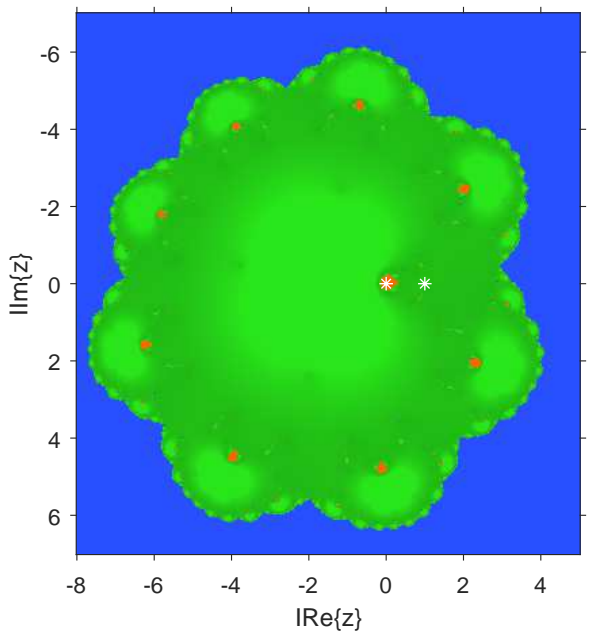

(a) $\beta=0.023-0.019 i$

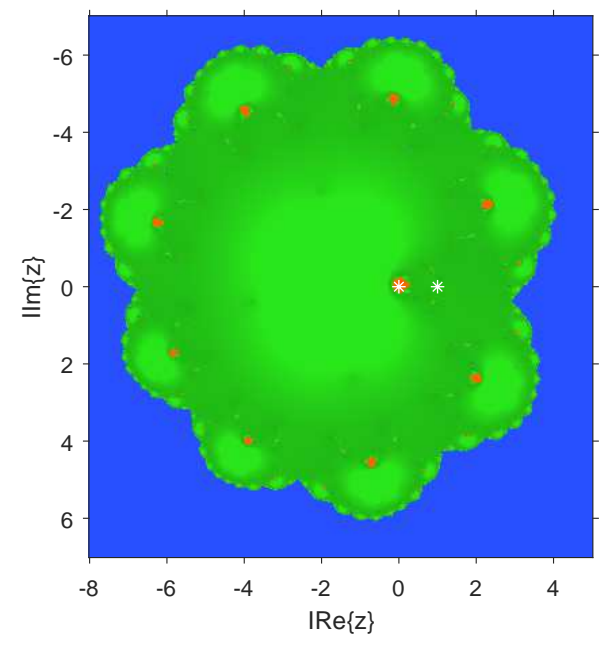

(b) $\beta=0.023+0.019 i$

Figure 8: Dynamical planes of $\beta=0.023-0.019 i$ and $\beta=0.023+0.019 i$.

\section{Numerical results}

In this section, we are going to apply some elements of family (??) to solve systems of nonlinear equations and use the obtained results from parameter and dynamical planes to apply the most stable members of the family for solving nonlinear problems and compare them with known eight order methods (??)-(??).

These results show that the selected methods of family (??) perform as well as known eight order methods, however the computations of the new method are less than other three methods. In each iteration of M7 we need to evaluate four times function $F$, once Jacobian and once a divided difference. But in $\mathrm{NM}_{8}, \mathrm{CCGT1}$ and CCGT2 one needs to evaluate two Jacobian and three functions per iteration. By using the approximated computational order of convergence (ACOC) introduced in [?] as

$$
p \approx A C O C=\frac{\ln \left(\left\|x^{(k+1)}-x^{(k)}\right\| /\left\|x^{(k)}-x^{(k-1)}\right\|\right)}{\ln \left(\left\|x^{(k)}-x^{(k-1)}\right\| /\left\|x^{(k-1)}-x^{(k-2)}\right\|\right)}
$$

we will check the theoretical order of convergence $p$ for the methods we test. 


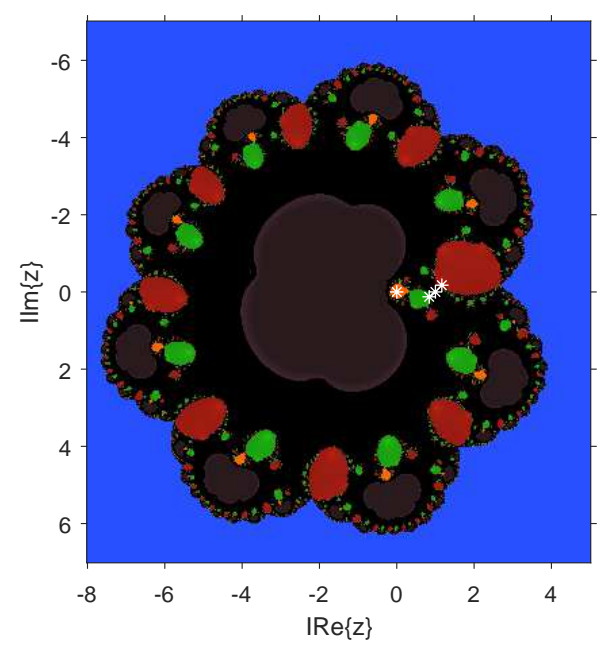

(a) $\beta=0.023-0.021 i$

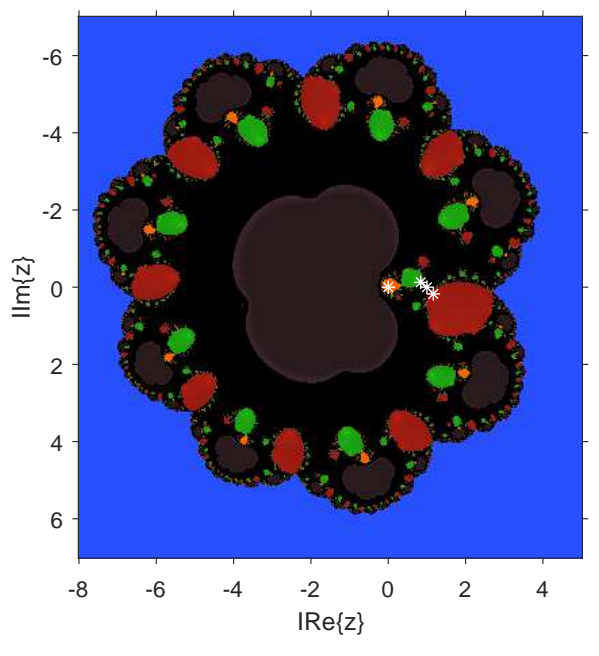

(b) $\beta=0.023+0.021 i$

Figure 9: Dynamical planes of $\beta=0.023-0.021 i$ and $\beta=0.023+0.021 i$.

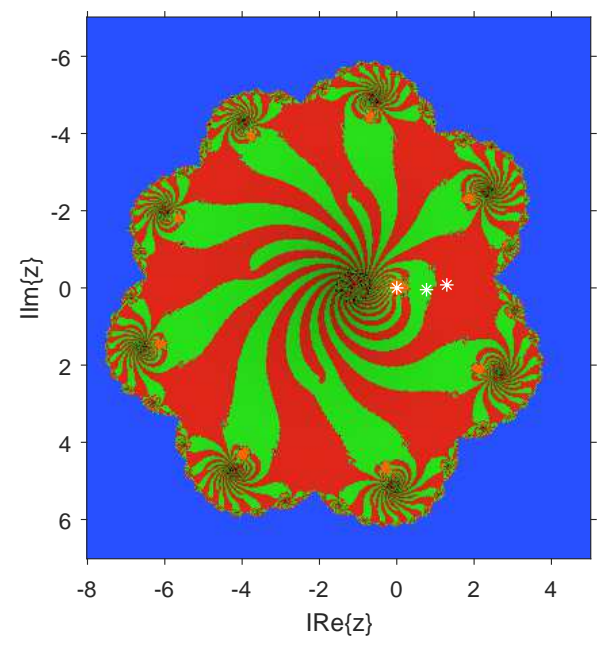

(a) $\beta=0.024-0.021 i$

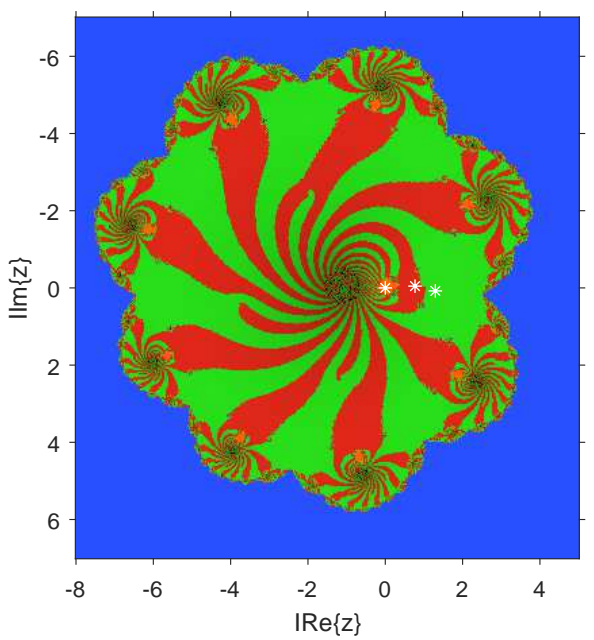

(b) $\beta=0.024+0.021 i$

Figure 10: Dynamical planes of $\beta=0.024-0.021 i$ and $\beta=0.024+0.021 i$.

Example 1. Let us consider the following nonlinear system

$$
x_{i}-\cos \left(2 x_{i}-\sum_{j=1}^{4} x_{j}\right)=0, \quad i=0,1,2, \ldots, n .
$$
$(1,1, \ldots, 1)$. In Table (??) we listed numerical results of this problem for some members of M7. All the experiments have been carried out in Matlab 2017 with precision arithmetics 10000 digits and the stopping criteria is $\left\|x^{(k+1)}-x^{(k)}\right\|<10^{-500}$ or $\left\|F\left(x^{(k+1)}\right)\right\|<10^{-500}$.

These results show that for the values of the parameter near 0 the method is unstable and in cases that

M7 is successful to solve the problem the order of convergence is not preserved. Also the member of the family corresponding to $\beta=0.2$ has the highest order of convergence. For other members far from the origin, the value of the approximated computational order of convergence is preserved and is not changed point by point, so the value of $\beta$ does not affect the order of convergence.

Comparing these results with those obtained for eight order methods (??)-(??), it can be observed that 


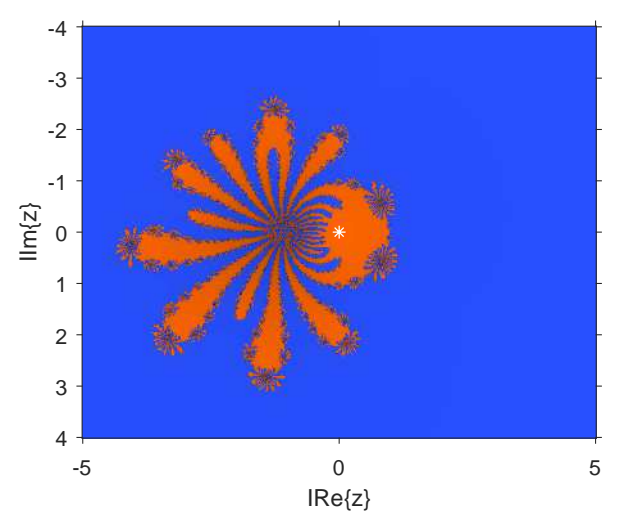

(a) $\beta=0.1-0.1 i$

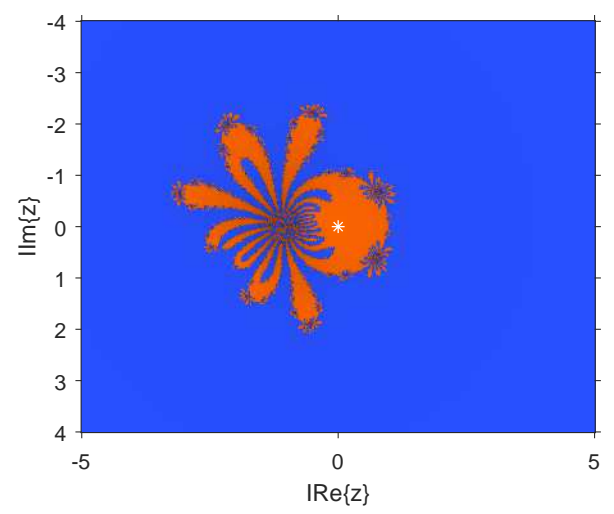

(c) $\beta=5+5 i$

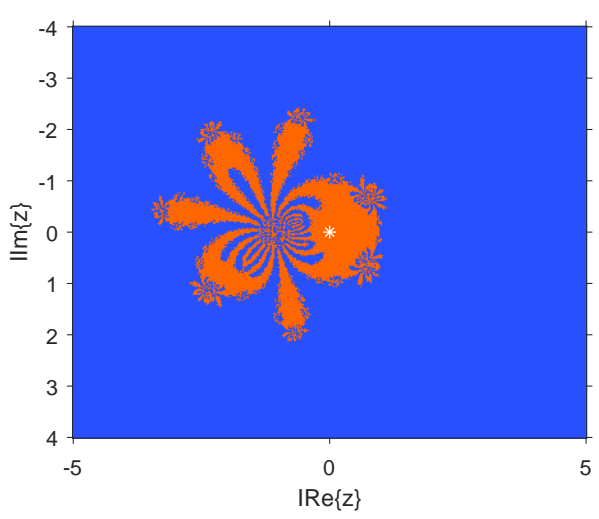

(b) $\beta=0.5+0.1 i$

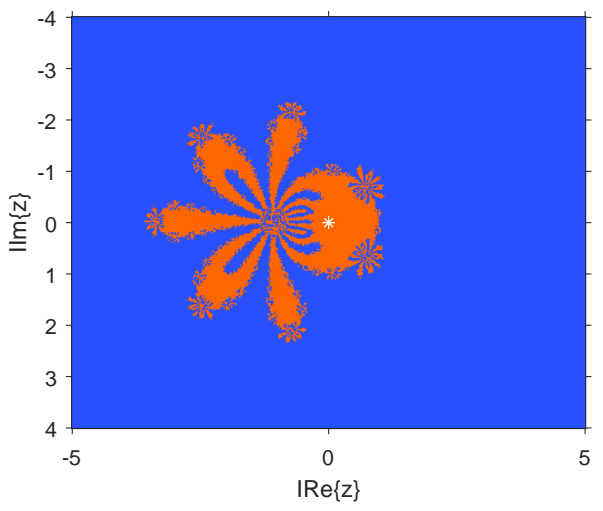

(d) $\beta=30+30 i$

Figure 11: Dynamical planes of $\beta=0.1-0.1 i, \beta=0.5+0.1 i, \beta=5+5 i$ and $\beta=30+30 i$.

\begin{tabular}{|c|c|c|c|c|c|}
\hline Method & parameter & iter & $\left\|x^{(k+1)}-x^{(k)}\right\|$ & $\left\|F\left(x^{(k+1)}\right)\right\|$ & ACOC \\
\hline \multirow{12}{*}{ M7 } & 0.001 & - & $\infty$ & $\infty$ & - \\
\hline & 0.005 & 6 & $1.31 \mathrm{e}-195$ & $4.89 \mathrm{e}-1364$ & 7.0 \\
\hline & 0.010 & 5 & $1.41 \mathrm{e}-344$ & $9.72 \mathrm{e}-2428$ & 7.0 \\
\hline & 0.019 & 4 & $1.47 \mathrm{e}-184$ & $1.14 \mathrm{e}-767$ & 4.0 \\
\hline & $0.023+0.021 \mathrm{i}$ & 5 & $5.30 \mathrm{e}-501$ & $1.36 \mathrm{e}-2033$ & 4.0 \\
\hline & $-0.039-0.028 \mathrm{i}$ & 5 & $9.10 \mathrm{e}-445$ & $4.32 \mathrm{e}-1843$ & 3.999 \\
\hline & $0.035-0.035 \mathrm{i}$ & 4 & $8.30 \mathrm{e}-180$ & $1.40 \mathrm{e}-1256$ & 7.0 \\
\hline & 0.2 & 4 & $3.38 \mathrm{e}-346$ & $2.10 \mathrm{e}-2770$ & 8.0 \\
\hline & 1 & 4 & $7.84 \mathrm{e}-333$ & $1.32 \mathrm{e}-2329$ & 7.0 \\
\hline & 2 & 4 & $9.75 \mathrm{e}-327$ & $8.23 \mathrm{e}-2287$ & 7.0 \\
\hline & 5 & 4 & $3.10 \mathrm{e}-304$ & $1.10 \mathrm{e}-2129$ & 7.0 \\
\hline & 20 & 4 & $8.13 \mathrm{e}-297$ & $3.0 \mathrm{e}-2077$ & 7.0 \\
\hline $\mathrm{NLM}_{8}$ & - & 4 & $1.65 \mathrm{e}-214$ & $1.99 \mathrm{e}-1711$ & 7.9999 \\
\hline CCGT1 & - & 4 & $5.23 \mathrm{e}-455$ & $1.68 \mathrm{e}-3638$ & 8.0 \\
\hline CCGT2 & - & 4 & $2.10 \mathrm{e}-297$ & $2.62 \mathrm{e}-2376$ & 8.0 \\
\hline
\end{tabular}

Table 1: Numerical results for Example 1.

the number of iterations to reach the convergence is the same, in spite of having one unit less in the order of convergence. So, in these cases we can conclude that our methods with vlues of the parameter far from the origin are more efficient. 


\begin{tabular}{|l||l|l|l|l|l|}
\hline Method & parameter & iter & $\left\|x^{(k+1)}-x^{(k)}\right\|$ & $\left\|F\left(x^{k+1}\right)\right\|$ & ACOC \\
\hline & 0.001 & - & $\infty$ & $\infty$ & - \\
& 0.005 & 4 & $2.92 \mathrm{e}-89$ & $3.39 \mathrm{e}-619$ & 7.0022 \\
M7 & 0.010 & 5 & $2.10 \mathrm{e}-359$ & $6.03 \mathrm{e}-2508$ & 7.0 \\
& 0.019 & 5 & $6.67 \mathrm{e}-127$ & $3.16 \mathrm{e}-535$ & 5.0507 \\
& $0.023+0.021 \mathrm{i}$ & 5 & $3.82 \mathrm{e}-182$ & $2.47 \mathrm{e}-756$ & - \\
& $-0.039-0.028 \mathrm{i}$ & 5 & $2.83 \mathrm{e}-236$ & $9.15 \mathrm{e}-974$ & - \\
& $0.035-0.035 \mathrm{i}$ & 4 & $5.0 \mathrm{e}-76$ & $1.82 \mathrm{e}-526$ & 6.9954 \\
& 0.2 & 4 & $2.97 \mathrm{e}-212$ & $2.04 \mathrm{e}-1693$ & 7.9999 \\
& 1 & 4 & $4.61 \mathrm{e}-151$ & $1.37 \mathrm{e}-1053$ & 6.9999 \\
& 2 & 4 & $5.44 \mathrm{e}-151$ & $4.34 \mathrm{e}-1053$ & 6.9999 \\
& 5 & 4 & $5.91 \mathrm{e}-149$ & $9.04 \mathrm{e}-1039$ & 6.9999 \\
& 20 & 4 & $6.38 \mathrm{e}-148$ & $1.66 \mathrm{e}-1031$ & 6.9999 \\
\hline NLM & -2 & 4 & $8.16 \mathrm{e}-369$ & $7.36 \mathrm{e}-2950$ & 8.0 \\
CCGT2 & - & 4 & $4.82 \mathrm{e}-380$ & $2.98 \mathrm{e}-3041$ & 8.0 \\
& - & 4 & $3.16 \mathrm{e}-376$ & $4.12 \mathrm{e}-3010$ & 8.0 \\
\hline
\end{tabular}

Table 2: Numerical results for Example 2.

Example 2. Let us consider the following nonlinear system

$$
\begin{aligned}
& x_{i}^{2} x_{i+1}-1=0, \quad i=0,1,2, \ldots, n-1, \\
& x_{n}^{2} x_{1}-1=0 . \quad i=n
\end{aligned}
$$

The solution of this problem is $\bar{x}=(1,1, \ldots, 1)^{T}$. We use $n=9$ and initial guess $x^{(0)}=(1.25,1.25, \ldots, 1.25)$.

In Table (??) we list the numerical results obtained in this this problem for some members of the M7. It can be observed that the performance of the methods is the same as in previous example.

\section{Conclusions}

In this manuscript, a new seventh-order family of parametric methods for solving nonlinear systems of equations is presented, with one element of order of convergence eight. As far as the authors know, there exist only a few methods with the same order of convergence and they are used in the numerical section for comparing with the proposed ones. The local order of convergence of the family is proved and the analysis of the stability of the whole family is performed by using techniques from complex discrete dynamics, showing a completely stable behavior, except by a very small region around $\beta=0$, where the iterative scheme is not defined. These analytical results have been checked by means of some dynamical planes and also with numerical tests on selected problems. In all cases, the members of the proposed family have shown a very stable performance.

[1] S. Amat, I. K. Argyros, S. Busquier, Á. A. Magreñán, Local convergence and the dynamics of a twopoint four parameter Jarratt-like method under weak conditions, Numerical Algorithms (2017), doi: $10.1007 / \mathrm{s} 11075-016-0152-5$.

[2] P. Blanchard, The dinamics of newton's method, Proc, Symp. Appl. math. 49 (1994) 139-154.

[3] P. Blanchard, Complex analytic dynamics on the riemann sphere, Bull. AMS 11 (1) (1984) 85-141 .

[4] F.I. Chicharro, A. Cordero, J.R. Torregrosa, Drawing dynamical and parameter planes of iterative families and methods. Sci. World J. 2013 (2013) 506-519.

[5] A. Cordero, J. García-Maimó, J.R. Torregrosa, M.P. Vassileva, P. Vindel, Chaos in King's iterative family, Appl. Math. Lett. 26 (2013) 842-848.

[6] A. Cordero, J. Garca-Maim, J.R. Torregrosa, M.P. Vassileva, Multidimensional stability analysis of a family of biparametric iterative methods, J. Math. Chem. 55 (2017) 1461-1480.

[7] A. Cordero, E. Gómez, J.R. Torregrosa, Efficient High-Order Iterative Methods for Solving Nonlinear Systems and Their Application on Heat Conduction Problems, Complexity 2017 (2017) Article ID 6457532, 11 pages.

[8] A. Cordero, J.M. Gutiérrez, Á. A. Magren, J.R. Torregrosa, Stability analysis of a parametric family of iterative methods for solving nonlinear models, Applied Mathematics and Computation 285 (2016) 26-40. 
[9] A. Cordero, F. Soleymani, J.R. Torregrosa, Dynamical analysis of iterative methods for nonlinear systems or how to deal with the dimension?, Applied Mathematics and Computation 244 (2014) 398-412.

[10] A. Cordero, J.R. Torregrosa, Variants of Newton's method using fifth-order quadrature formulas, Appl. Math. Comput. 190 (2007) 686-698.

[11] Y.H. Geum, Y.I. Kim, B. Neta, A sixth-order family of three-point modified Newton-like multiple-root finders and the dynamics behind their extraneous fixed points, Appl. Math. Comput. 283 (2016) 120-140.

[12] J.M. Gutiérrez, M.A. Hernández, N. Romero, Dynamics of a new family of iterative processes for quadratic polynomials, J. Comput. Appl. Math. 233 (2010) 2688-2695.

[13] J.M. Gutiérrez, S. Plaza, N. Romero, Dynamics of a fifth-order iterative method, Intern. J. Comput. Math., 89(6) (2012) 822-835.

[14] Á. A. Magreñán, Different anomalies in a Jarratt family of iterative root-finding methods, Applied Mathematics and Computation 233 (2014) 29-38.

[15] J.M. Ortega, W.C. Rheinboldt, Iterative Solution of Nonlinear Equations in Several Variables, Academic Press, 1970.

[16] A.M. Ostrowski, Solution of the equations and systems of equations, Prentice-Hall, England Cliffs, New Yourk, 1964.

[17] J.R. Sharma, H. Arora, Improved Newton-like methods for solving systems of nonlinear equations, SeMA Journal 74(2) (2017) 147-163. 